\title{
A new parameterization for waveform inversion in acoustic orthorhombic media
}

\author{
Nabil Masmoudi ${ }^{1}$ and Tariq Alkhalifah ${ }^{1}$
}

\begin{abstract}
Orthorhombic anisotropic model inversion is extra challenging because of the multiple parameter nature of the inversion problem. The high number of parameters required to describe the medium exerts considerable trade-off and additional nonlinearity to a full-waveform inversion (FWI) application. Choosing a suitable set of parameters to describe the model and designing an effective inversion strategy can help in mitigating this problem. Using the Born approximation, which is the central ingredient of the FWI update process, we have derived radiation patterns for the different acoustic orthorhombic parameterizations. Analyzing the angular dependence of scattering (radiation patterns) of the parameters of different parameterizations starting with the often used Thomsen-Tsvankin parameterization, we have assessed the potential trade-off between the parameters and the resolution in describing the data and inverting for the parameters. The analysis led us to
\end{abstract}

introduce new parameters $\epsilon_{d}, \delta_{d}$, and $\eta_{d}$, which have azimuthally dependent radiation patterns, but keep the scattering potential of the transversely isotropic parameters stationary with azimuth (azimuth independent). The novel parameters $\epsilon_{d}, \delta_{d}$, and $\eta_{d}$ are dimensionless and represent a measure of deviation between the vertical planes in orthorhombic anisotropy. Therefore, these deviation parameters offer a new parameterization style for an acoustic orthorhombic medium described by six parameters: three vertical transversely isotropic (VTI) parameters, two deviation parameters, and one parameter describing the anisotropy in the horizontal symmetry plane. The main feature of any parameterization based on the deviation parameters, is the azimuthal independency of the modeled data with respect to the VTI parameters, which allowed us to propose practical inversion strategies based on our experience with the VTI parameters. This feature of the new parameterization style holds for even the long-wavelength components of the model constrained by traveltimes.

\section{INTRODUCTION}

Wide-azimuth and wide-aperture seismic surveys reveal that anisotropy has a clear footprint on the data we observe and as a consequence, more reliable imaging and inversion algorithms should preserve at least the most effective anisotropy behavior of the subsurface. Numerous studies show that taking into account anisotropy in modeling and inversion algorithms yields better results (Barnes et al., 2008; Prieux et al., 2011; Wang et al., 2013).

Modeling the complexity of the subsurface physics is a challenge. Although elastic wavefield modeling allows realistic physics and a good description of the wave dynamics, it is computationally expensive and is rarely affordable for current 3D real data applications. On the other hand, acoustic wavefield modeling is cheaper, more efficient, and provides an accurate description of the P-waves kinematics (Alkhalifah, 2003). Decreasing the number of parameters to be inverted is another advantage of the acoustic approximation. However, the dynamics are not well-represented in acoustic modeling, albeit the acoustic assumption has been used in fullwaveform analysis (Alkhalifah and Plessix, 2014) and inversion (Cheng et al., 2014). To handle the acoustic misrepresentation of the amplitudes, it is a common practice to use the density to improve the data fit for an imperfect amplitude match due to elastic effects (Prieux et al., 2013).

Some successful model parameter inversions have been applied under the vertical transversely isotropic (VTI) media assumption. For instance, Operto et al. (2015) apply 3D frequency-domain full-waveform inversion (FWI) (Pratt, 1999) on real offshore seis-

\footnotetext{
Manuscript received by the Editor 15 November 2015; revised manuscript received 10 February 2016; published online 26 May 2016.

${ }^{1}$ King Abdullah University of Science and Technology (KAUST), Physical Science and Engineering Division, Thuwal, Saudi Arabia. E-mail: nabil. masmoudi@kaust.edu.sa; tariq.alkhalifah@kaust.edu.sa.

(C) 2016 Society of Exploration Geophysicists. All rights reserved.
} 
mic data using the visco-acoustic VTI approximation. They update the vertical velocity during their inversion, while keeping density, attenuation, and Thomsen anisotropy parameters unchanged. Furthermore, Wang et al. (2012) show 3D real data inversion examples, in which they account for anisotropy in waveform simulation but invert only for the vertical velocity. Moreover, Cheng et al. (2014) apply a hierarchical approach on real data by updating the vertical velocity; first, keeping the anisotropy parameters unchanged and switching to joint inversion when vertical velocity inversion is approaching convergence. On the other hand, da Silva et al. (2014) propose a simultaneous inversion in which they update the vertical and horizontal velocities simultaneously. Behind all these inversion strategies lies a need for understanding the resolvability, resolution limits, and trade-off between the parameters. Indeed, one of the main issues in a multiparameter inversion problem is that velocity and anisotropy coefficients have different physical units and very different influences on surface seismic data. This behavior is intriguing because the influence of one parameter on the modeled data depends on the other parameters involved in the parameterization. Therefore, identifying a suitable parameterization and analyzing which parameters in the chosen parameterization can be reliably resolved is at the heart of any multiparameter inversion application.

To analyze the dependency of the data on different parameterization classes, one should understand the angular influence of the perturbed parameters, specifically, the radiation patterns. Such analysis is presented for elastic media in Wu and Aki (1985), Forgues and Lambaré (1997), and Burridge et al. (1998) and for different acoustic VTI parameterizations in Gholami et al. (2013a, 2013b) and Alkhalifah and Plessix (2014). Another approach based on the decomposition of the Hessian matrix (Kiyashchenko et al., 2004) is proposed to analyze acoustic VTI parameters. One can also study the finite-frequency sensitivity kernels as shown in Djebbi and Alkhalifah (2014) for multicomponent VTI media. In a nutshell, these analyses reveal the critical issues of a multiparameter inversion and pave the road to build better strategies to tackle inversion problems in anisotropic media.

In fact, for 3D surface seismic data with clear azimuthal dependency due to subsurface fractures, relying on a VTI model representation is not realistic and the need to account for lower symmetries such as orthorhombic is necessary (Wild and Crampin, 1991; Schoenberg and Helbig, 1997; Tsvankin et al., 2010). In this paper, we focus on orthorhombic anisotropy and address the fundamental issues of a suitable orthorhombic model parameterization in its simplest acoustic representation. We exclude density and attenuation from this study and assume that the elastic effects on the amplitudes can be approximately included in the short wavelength variations of one of the acoustic parameters. Thus, our conclusions are more suitable to inversion relying on fitting the phase of the data.

Table 1. Different parameterizations for an acoustic orthorhombic media.

Type 1

parameterization

Type 2

parameterization

Type 3

\begin{tabular}{ccc}
\hline$\left(\mathbf{v}_{v}, \boldsymbol{\delta}_{1}, \boldsymbol{\delta}_{2}, \mathbf{\epsilon}_{1}, \mathbf{\epsilon}_{2}, \boldsymbol{\delta}_{3}\right)$ & $\left(\mathbf{v}_{v}, \mathbf{v}_{n 1}, \mathbf{v}_{n 2}, \boldsymbol{\eta}_{1}, \boldsymbol{\eta}_{2}, \boldsymbol{\delta}_{3}\right)$ & $\left(\mathbf{v}_{v}, \mathbf{v}_{n 1}, \mathbf{v}_{n 2}\right.$, \\
$\left.\mathbf{v}_{h 1}, \mathbf{v}_{h 2}, \boldsymbol{\delta}_{3}\right)$ \\
$\left(v_{v}, \eta_{1}, \eta_{2}, \epsilon_{1}, \epsilon_{2}, \delta_{3}\right)$ & $\left(v_{v}, v_{h 1}, v_{h 2}, \eta_{1}, \eta_{2}, \delta_{3}\right)$ & - \\
$\left(v_{v}, \delta_{1}, \delta_{2}, \eta_{1}, \eta_{2}, \delta_{3}\right)$ & $\left(v_{v}, v_{h 1}, v_{h 2}, \delta_{1}, \delta_{2}, \delta_{3}\right)$ & - \\
\hline
\end{tabular}

Based on the Born approximation and a weak local perturbation in a generally constant or a smooth isotropic background, we derive the radiation perturbation patterns for different classes of orthorhombic parameterization. We first base our parameterization investigation on the conventional Thomsen-Tsvankin notation style. Through the radiation patterns, we analyze the sensitivity of the data to each parameter class and pinpoint the potential trade-off between the parameters and the subsequent resolvability issues. To handle the considerable number of parameters in an orthorhombic inversion problem, we propose practical strategies based on a new parameterization style. Our analysis is supplemented with a study of the first-order sensitivity of the traveltimes with respect to the perturbations induced by the different model parameters.

\section{RADIATION PATTERNS OF CONVENTIONAL ORTHORHOMBIC PARAMETERIZATIONS}

At the heart of the FWI update process is the gradient of the FWI objective functional with respect to the inverted parameters. The gradient is conveniently described by the Born approximation (Cohen and Bleistein, 1977; Panning et al., 2009). This gradient maps the sensitivity of the data to perturbations in the velocity model, and thus, allows us to map data residuals to their corresponding perturbation locations in the model. On the other hand, the proper amplitude of such model perturbation is usually given by the second-order dependency of the objective functional on the parameters (referred to as the Hessian). To develop the sensitivity of the objective function to the model parameters, we have to first decide on the parameterization of our orthorhombic model.

\section{Different types of acoustic orthorhombic parameterization}

An orthorhombic medium, under the acoustic approximation, can be parametrized by six anisotropy parameters without considering the density or attenuation. Among the possible parameterizations, we identify three types of parameterization as shown in Table 1 . The first one is referred to as type 1 parameterization, and it involves one velocity and five dimensionless parameters. The second type is referred to as type 2 parameterization, and involves three velocities and three dimensionless parameters. The last type, referred to as type 3 parameterization, involves five velocities and one dimensionless parameter. In the identified parameter classes (Table 1), $v_{v}$ represents the P-wave vertical velocity; $v_{n 1}$ and $v_{n 2}$ are the normal moveout (NMO) velocities in the $[x, z]$ - and $[y, z]$-symmetry planes, respectively; $v_{h 1}$ and $v_{h 2}$ are the horizontal velocities in the $[x, z]$ and $[y, z]$-symmetry planes, respectively; $\epsilon_{1}, \delta_{1}$, and $\eta_{1}$ are defined in the $[x, z]$-symmetry plane; $\epsilon_{2}, \delta_{2}$, and $\eta_{2}$ are defined in the $[y, z]$-symmetry plane; and $\delta_{3}$ describes the anisotropy in the horizontal symmetry plane $[x, y]$, and is defined with respect to the $x$-coordinate axis. The relations between these parameters are as follows (Tsvankin, 1997; Alkhalifah, 2003):

$$
\begin{aligned}
v_{n 1} & =v_{v} \sqrt{1+2 \delta_{1}}, \quad v_{n 2}=v_{v} \sqrt{1+2 \delta_{2}}, \\
v_{h 1} & =v_{n 1} \sqrt{1+2 \eta_{1}}, \quad v_{h 2}=v_{n 2} \sqrt{1+2 \eta_{2}}, \\
\eta_{1} & =\frac{\epsilon_{1}-\delta_{1}}{1+2 \delta_{1}}, \quad \eta_{2}=\frac{\epsilon_{2}-\delta_{2}}{1+2 \delta_{2}} .
\end{aligned}
$$


In the identified parameter classes, note that the vertical velocity is a necessary parameter required in each parameterization. This constraint reduces the number of possible orthorhombic parameterization. The dimensionless parameter $\delta_{3}$ is also a recurrent parameter and $\delta_{3}$ can be replaced by another parameter, such as $\eta_{3}$ (Grechka and Tsvankin, 1999) or $\eta_{x y}$ (Stovas, 2015).

\section{Deriving the radiation patterns}

We derive the radiation patterns for different orthorhombic parameterizations using the Born approximation and the acoustic wave equation (Alkhalifah, 2003). In this derivation, we choose a parameterization with the three velocities $v_{v}, v_{n 1}$, and $v_{n 2}$, and the three dimensionless parameters $\eta_{1}, \eta_{2}$, and $\chi$, where $\chi$ is related to $\delta_{3}$ through the relation $\chi=\sqrt{1+2 \delta_{3}}$, and introduced here to simplify the derivation. Later, we use the relations between the parameters to obtain the radiation patterns for different parameterizations.

The acoustic wave equation for an orthorhombic medium in the frequency domain can be written as (Alkhalifah, 2003)

$$
\begin{gathered}
-\omega^{6} P-A \omega^{4} \frac{\partial^{2} P}{\partial x^{2}}-B \omega^{4} \frac{\partial^{2} P}{\partial y^{2}}-C \omega^{4} \frac{\partial^{2} P}{\partial z^{2}}+D \omega^{2} \frac{\partial^{4} P}{\partial x^{2} \partial y^{2}} \\
+E \omega^{2} \frac{\partial^{4} P}{\partial x^{2} \partial z^{2}}+F \omega^{2} \frac{\partial^{4} P}{\partial y^{2} \partial z^{2}}-G \frac{\partial^{6} P}{\partial x^{2} \partial y^{2} \partial z^{2}}=S(w),
\end{gathered}
$$

where $P$ is the pressure field in the frequency domain at a location $(x, y, z), S(\omega)$ is the source term, and the coefficients $A, B, C, D, E$, $F$, and $G$ are related to the orthorhombic parameters through these relations (Ibanez-Jacome et al., 2014)

$$
\begin{aligned}
A= & v_{n 1}^{2}\left(1+2 \eta_{1}\right), \quad B=v_{n 2}^{2}\left(1+2 \eta_{2}\right), \quad C=v_{v}^{2}, \\
D= & v_{n 1}^{2}\left(1+2 \eta_{1}\right)\left(\left(1+2 \eta_{1}\right) \chi^{2} v_{n 1}^{2}-\left(1+2 \eta_{2}\right) v_{n 2}^{2}\right), \\
E= & -2 \eta_{1} v_{n 1}^{2} v_{v}^{2}, \quad F=-2 \eta_{2} v_{n 2}^{2} v_{v}^{2}, \\
G= & -v_{v}^{2} v_{n 1}^{2}\left(\left(1+2 \eta_{1}\right)^{2} v_{n 1}^{2} \chi^{2}-2 \chi\left(1+2 \eta_{1}\right) v_{n 1} v_{n 2}\right. \\
& \left.+v_{n 2}^{2}\left(1-4 \eta_{1} \eta_{2}\right)\right) .
\end{aligned}
$$

In equation 2 , we assume that the symmetry planes of the orthorhombic medium coincide with the coordinate planes.

To derive the Born approximation, we define the perturbations $r_{v_{n 1}^{2}}, r_{v_{n 2}^{2}}, r_{v_{v}^{2}}, r_{\eta_{1}}, r_{\eta_{2}}$, and $r_{\chi^{2}}$, such as

$$
\begin{array}{cc}
v_{n 1}^{2}=v_{n 1_{0}}^{2}\left(1+r_{v_{n 1}^{2}}\right), & v_{2}^{2}=v_{n 2_{0}}^{2}\left(1+r_{v_{n 2}^{2}}\right), \\
v_{v}^{2}=v_{v_{0}}^{2}\left(1+r_{v_{v}^{2}}\right), & \eta_{1}=\eta_{1_{0}}\left(1+r_{\eta_{1}}\right), \\
\eta_{2}=\eta_{2_{0}}\left(1+r_{\eta_{2}}\right), & \chi^{2}=\chi_{0}^{2}\left(1+r_{\chi^{2}}\right),
\end{array}
$$

where the subscript 0 denotes the model parameters in the background medium. We define $P_{0}$, the pressure field in the background medium. In this case, $P_{0}$ satisfies the following wave equation:

$$
\mathcal{L} P_{0}=S,
$$

where $\mathcal{L}$ is the orthorhombic wave equation operator for the background medium. We replace the perturbations, defined in equation 4 , into the wave equation 2 and obtain an additional source term $S_{v}(P)$, usually referred to as a virtual source

$$
\mathcal{L} P=S+S_{v}(P)
$$

The wavefield $P$ can be decomposed into a background wavefield $P_{0}$ and a scattered wavefield $P_{s}$

$$
P=P_{0}+P_{s}
$$

Using equation 5 and the Born approximation (which induces a scattered wavefield $P_{s}$ negligible compared with the background wavefield $P_{0}$ ) yields

$$
\mathcal{L} P_{s}\left(\mathbf{x}, \mathbf{x}_{s}, \omega\right)=S_{v}\left(P_{0}\right)
$$

By defining the Green's function $G_{r}$ of the receivers as follows:

$$
\mathcal{L} G_{r}\left(\mathbf{x}, \mathbf{x}_{r}, \omega\right)=-\delta\left(\mathbf{x}-\mathbf{x}_{r}\right),
$$

and using Green's theorem, the scattered wavefield $P_{s}$ in the farfield can be obtained

$$
P_{s}\left(\mathbf{x}_{s}, \mathbf{x}_{r}, \omega\right)=-\int_{0}^{\infty} S_{v} G_{r} d \mathbf{x}
$$

We assume an isotropic background for simplicity of analysis $\left(\eta_{1_{0}}=0, \eta_{2_{0}}=0, \chi_{0}=1, v_{n 1}=v_{n 2}=v_{v}=v_{0}\right)$, which is an assumption generally used for such derivation (Alkhalifah and Plessix, 2014), and we obtain the scattered wavefield $P_{S}$ in this form

$$
P_{s}=\omega^{2} v_{0}^{2} \int_{0}^{\infty}\left(\begin{array}{c}
\omega^{2} \frac{\partial P_{0}}{\partial x} \frac{\partial G_{r}}{\partial x}+v_{0}^{2} \frac{\partial^{2} P_{0}}{\partial x \partial y} \frac{\partial^{2} G_{r}}{\partial x \partial y} \\
\omega^{2} \frac{\partial P_{0}}{\partial y} \frac{\partial G_{r}}{\partial y}-v_{0}^{2} \frac{\partial^{2} P_{0}}{\partial x \partial y} \frac{\partial^{2} G_{r}}{\partial x \partial y} \\
\omega^{2} \frac{\partial P_{0}}{\partial z} \frac{\partial G_{r}}{\partial z} \\
2\left(\omega^{2} \frac{\partial P_{0}}{\partial x} \frac{\partial G_{r}}{\partial x}+v_{0}^{2} \frac{\partial^{2} P_{0}}{\partial x \partial y} \frac{\partial^{2} G_{r}}{\partial x \partial y}-v_{0}^{2} \frac{\partial^{2} P_{0}}{\partial x \partial z} \frac{\partial^{2} G_{r}}{\partial x \partial z}\right) \\
2\left(\omega^{2} \frac{\partial P_{0}}{\partial y} \frac{\partial G_{r}}{\partial y}-v_{0}^{2} \frac{\partial^{2} P_{0}}{\partial x \partial y} \frac{\partial^{2} G_{r}}{\partial x \partial y}-v_{0}^{2} \frac{\partial^{2} P_{0}}{\partial y \partial z} \frac{\partial^{2} G_{r}}{\partial y \partial z}\right) \\
v_{0}^{2} \frac{\partial^{2} P_{0}}{\partial x \partial y} \frac{\partial^{2} G_{r}}{\partial x \partial y} \\
r_{v_{n 1}^{2}} \\
r_{v_{v}^{2}} \\
r_{\eta_{1}} \\
r_{\eta_{2}} \\
r_{\chi^{2}}
\end{array}\right) d \mathbf{x .}
$$

Then, we replace the background wavefield $P_{0}$ and the receiver (scattered) wavefield by the asymptotic (planar) Green's functions $G_{s}$ and $G_{r}$, respectively, as follows:

$$
\begin{aligned}
& G_{s}(\mathbf{x}, \omega)=A_{s} \exp \left(-i \omega \frac{\mathbf{p}_{\mathbf{s}} \cdot \mathbf{x}}{v_{0}}\right), \\
& G_{r}(\mathbf{x}, \omega)=A_{r} \exp \left(-i \omega \frac{\mathbf{p}_{\mathbf{r}} \cdot \mathbf{x}}{v_{0}}\right),
\end{aligned}
$$


where $A_{s}$ and $A_{r}$ are the amplitudes of the Green's functions, $\mathbf{p}_{\mathbf{s}}$ and $\mathbf{p}_{\mathbf{r}}$ are unitary vectors defined at the scatterer point, and represent the direction of the incident and reflected wave, respectively. We choose these conventions for $\mathbf{p}_{\mathbf{s}}$ and $\mathbf{p}_{\mathbf{r}}$ :

$$
\begin{gathered}
\mathbf{p}_{\mathbf{s}}=\left(\begin{array}{c}
p_{s x}=\sin \theta \cos \phi \\
p_{s y}=\sin \theta \sin \phi \\
p_{s z}=\cos \theta
\end{array}\right), \\
\mathbf{p}_{\mathbf{r}}=\left(\begin{array}{c}
p_{r x}=-\sin \theta \cos \phi \\
p_{r y}=-\sin \theta \sin \phi \\
p_{r z}=\cos \theta
\end{array}\right),
\end{gathered}
$$

where $\theta$ and $\phi$ are the incident angle and the source-to-receiver azimuth angle, respectively (see Figure 1). Substituting equation 13 into equation 11 , we obtain the scattered field

$$
P_{s}=\omega^{6} \int G_{s}\left(\mathbf{x}_{s}, \mathbf{x}, \omega\right) G_{r}\left(\mathbf{x}_{r}, \mathbf{x}, \omega\right) a_{0}(\mathbf{x}) \cdot r_{0}(\mathbf{x}) d x,
$$

where

$$
\begin{aligned}
a_{0}(\mathbf{x})= & \left(\begin{array}{c}
p_{s x} p_{r x}\left(p_{s y} p_{r y}-1\right) \\
p_{s y} p_{r y}\left(-p_{s x} p_{r x}-1\right) \\
-p_{s z} p_{r z} \\
-2 p_{s x} p_{r x}\left(1-p_{s y} p_{r y}+p_{s z} p_{r z}\right) \\
-2 p_{s y} p_{r y}\left(1+p_{s x} p_{r x}+p_{s z} p_{r z}\right) \\
r_{0}(\mathbf{x})= \\
p_{s x} p_{s y} p_{r x} p_{r y} \\
r_{v_{v}^{2}} \\
r_{\eta_{1}} \\
r_{\eta_{2}} \\
r_{\chi^{2}}
\end{array}\right),
\end{aligned}
$$

where the vector $a_{0}(\mathbf{x})$ defines the radiation patterns for the perturbation parameters $r_{v_{n 1}^{2}}, r_{v_{n 2}^{2}}, r_{v_{v}^{2}}, r_{\eta_{1}}, r_{\eta_{2}}$, and $r_{\chi^{2}}$. We emphasize that

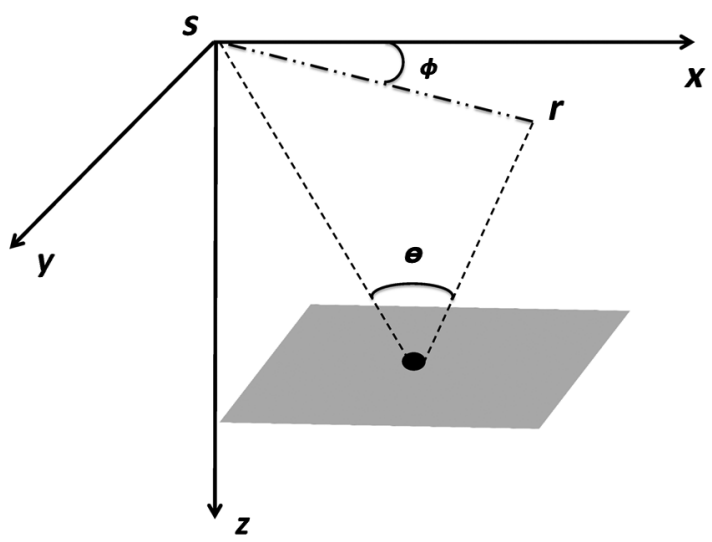

Figure 1. A schematic plot of the source and receiver rays as they connect at model point (black spot) and the angles involved. the procedure described above assumes locally (around the scatterer) constant or smooth background, which is the same assumption behind the Born linearized approximation.

To derive the radiation patterns for any parameterization, one can write the scattered field as follows:

$$
P_{s}=\omega^{6} \int G_{s}\left(\mathbf{x}_{s}, \mathbf{x}, \omega\right) G_{r}\left(\mathbf{x}_{r}, \mathbf{x}, \omega\right) a_{i}(\mathbf{x}) \cdot r_{i}(\mathbf{x}) d x,
$$

with $a_{i}$ and $r_{i}$ having components that depend on the parameterization choice. Therefore, as shown in Appendix A, we obtain the scattered wavefield for the parameterizations presented in this paper by using the relations between the parameters and the perturbations.

\section{Interpretation of the radiation patterns}

We study the radiation patterns for the parameterizations highlighted in bold in Table 1. The remaining four parameterizations have similar behavior to those we show here, in terms of azimuthal dependency of the radiation patterns of the different parameters.

\section{Type 1 parameterization: One velocity + five dimensionless parameters}

Figure 2 shows the radiation patterns for the parameterization $\left(v_{v}, \epsilon_{1}, \epsilon_{2}, \delta_{1}, \delta_{2}, \delta_{3}\right)$ as a function of the scattering angle for different azimuth angles. As shown in Figure 2a, the vertical velocity has angles (scattering and azimuth angles) invariant radiation pattern, similar to the isotropic case. The parameters $\epsilon_{1}$ and $\epsilon_{2}$ are sensitive to large scattering angles (Figure $2 \mathrm{~d}$ and 2e), whereas $\delta_{1}$ and $\delta_{2}$ are associated with intermediate angles (Figure $2 \mathrm{~b}$ and $2 \mathrm{c}$ ). Moreover, the maximum scattering energy of $\delta_{1}$ and $\epsilon_{1}$ is at $\phi=0^{\circ}$, whereas for $\delta_{2}$ and $\epsilon_{2}$ is at $\phi=90^{\circ}$. This is expected because the parameters $\left(\epsilon_{1}, \delta_{1}\right)$ and $\left(\epsilon_{2}, \delta_{2}\right)$ are defined in the symmetry planes $[x, z]$ and $[y, z]$, respectively. Finally, the scattering potential of $\delta_{3}$ (Figure 2f) is associated with large scattering angles, and it is maximum when $\phi=45^{\circ}$.

The advantage of this parameterization is the limited trade-off between $\delta_{1}$ and $\epsilon_{1}$ as well as between $\delta_{2}$ and $\epsilon_{2}$. In this case, data from large scattering angles and different azimuths are needed to update $\epsilon_{1}$ and $\epsilon_{2}$, whereas data from intermediate angles could be used to update $\delta_{1}$ and $\delta_{2}$. This is granted that the vertical velocity is known from, for example, a well. Indeed, note the trade-off between the vertical velocity and the parameters $\epsilon_{1}$ and $\epsilon_{2}$ at large scattering angles but not at short angles. This means that the long wavelength information of the velocity is not uniquely constrained by diving waves and trade-off occurs at practically all azimuths. In general, unless the vertical velocity is constrained using well logs, the long wavelength information for the vertical velocity will not be updated properly. In this parameterization, using diving waves, there is a trade-off between the vertical velocity and $\epsilon_{1,2}$ for the long wavelength information, and using tomography or migration velocity analysis, there is a trade-off between the vertical velocity and $\delta_{1,2}$ for such long wavelength information.

Type 2 parameterization: Three velocities + three dimensionless parameters

Figure 3 shows the radiation patterns for the parameterization $\left(v_{n 1}, v_{n 2}, v_{v}, \eta_{1}, \eta_{2}, \delta_{3}\right)$ as a function of the scattering angle and different azimuth angles. Figure $3 b-3$ f shows that the scattering po- 
tential of $v_{n 1}, v_{n 2}$ as well as $\eta_{1}, \eta_{2}$, and $\delta_{3}$ is maximum at large scattering angles. These parameters also effect the data at different azimuths. Moreover, the radiation pattern of the vertical velocity $v_{v}$ (Figure 3a) is azimuthally independent and is associated with vertically traveling waves (corresponding to small scattering angles).

From these observations, we conclude that, the vertical velocity is not resolvable from diving waves, the main source of long wavelength information, and we can only update the scattering $v_{v}$ to fit the amplitude at short offsets. Also, there is a trade-off between $v_{n 1}$ and $\eta_{1}$ as well as between $v_{n 2}$ and $\eta_{2}$ at large scattering angles, which cannot be resolved with small and intermediate scattering angles data. Finally, $\delta_{3}$ is weakly resolvable due to the trade-off at $45^{\circ}$ azimuth and large scattering angles between the different parameters. Overall, this parameterization induces considerable crosstalk between many parameters and is not suitable for wavefield inversion.

\section{Type 3 parameterization: Five velocities + one dimensionless parameter}

Figure 4 shows the radiation patterns for the parameterization with five velocities and one dimensionless parameter $\left(v_{h 1}, v_{h 2}\right.$, $\left.v_{n 1}, v_{n 2}, v_{v}, \delta_{3}\right)$. In this case, the scattering potential of the horizontal velocities is associated with large scattering angles, as shown in Figure $4 \mathrm{~d}$ and $4 \mathrm{e}$. In addition, the main influences of $v_{h 1}$ and $v_{h 2}$ are observed at different azimuths. Moreover, Figure $4 \mathrm{~b}$ and $4 \mathrm{c}$ shows that the NMO velocities radiate at intermediate scattering angles, and have no influence on the vertical and horizontal wave paths. As to the vertical velocity $v_{v}$ (Figure $4 \mathrm{a}$ ), it has an azimuthally independent radiation patterns associated with vertically traveling wave paths. The behavior of $\delta_{3}$ (Figure $4 \mathrm{f}$ ) in this parameterization is similar to the one observed in the previous two cases.

An inversion procedure based on this parameterization can start from diving waves, which allow to update the horizontal velocities. Then, by including intermediate scattering angles and using different azimuths, one might be able to update the NMO velocities. Still, we expect this strategy to converge to only low-resolution (smooth) velocity models. Moreover, it might be appealing to set an inversion with five velocities that have the same units to avoid relying on the Hessian for scaling. However, the sensitivity of the data to these velocities varies considerably with offsets and azimuths, and thus, the Hessian is still required to scale such variations, otherwise, if the horizontal velocities are strongly updated, whereas the vertical velocity is kept stationary, the resulting anisotropy will be erratic. That is why dealing with one velocity and five dimensionless parameters is more convenient. A smooth dimensionless parameter can represent very detailed velocity model if this parameter is combined with a complex velocity model. In other words, an inversion that results in a smooth dimensionless parameter and an accurate a)

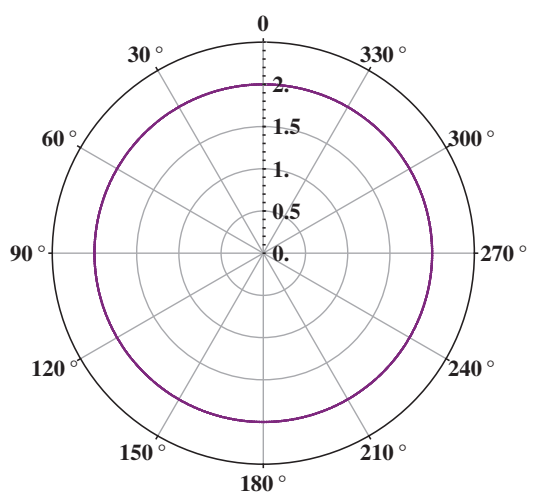

d)

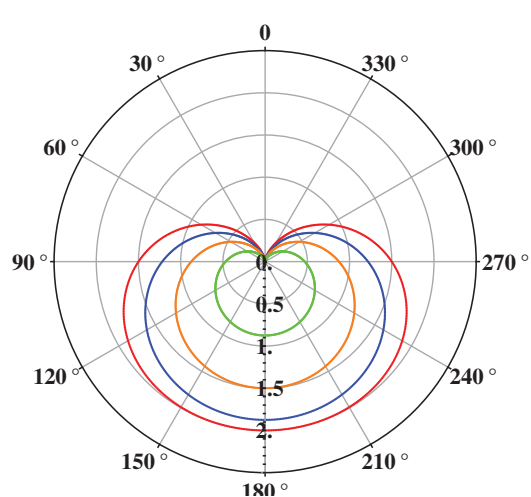

b)

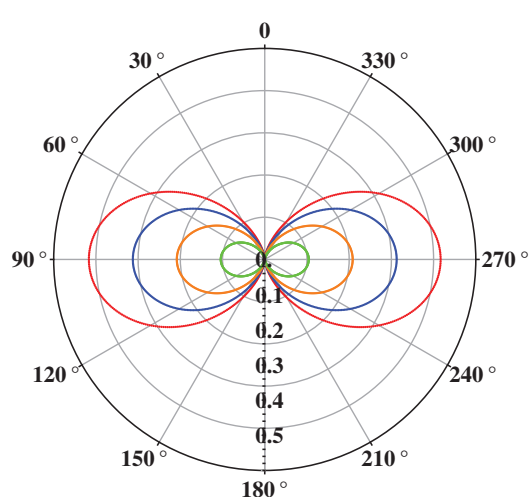

e)

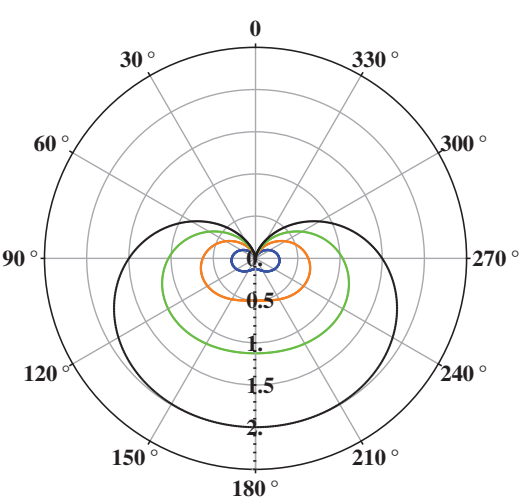

c)

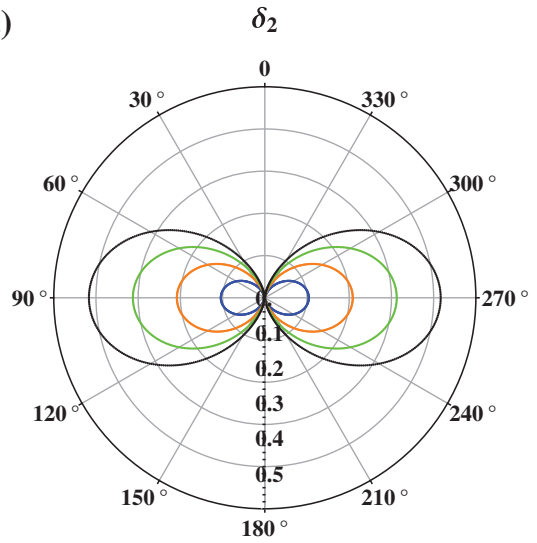

f)

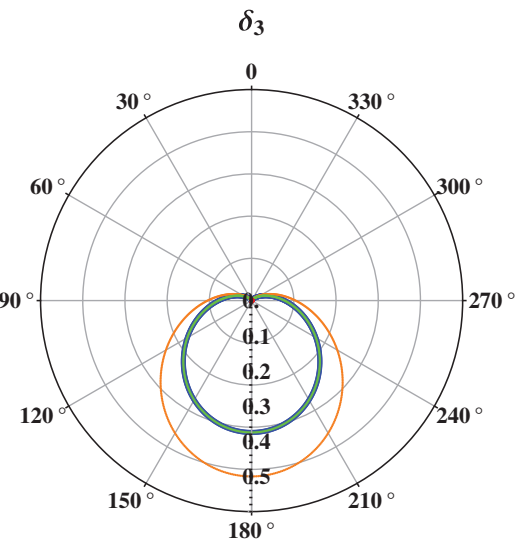

Figure 2. Radiation patterns for the parameterization with $\left(v_{v}, \delta_{1}, \delta_{2}, \epsilon_{1}, \epsilon_{2}, \delta_{3}\right)$ as a function of the scattering angle for different azimuth angles $\phi: 0^{\circ}$ (red), $30^{\circ}$ (blue), $45^{\circ}$ (orange), $60^{\circ}$ (green), $90^{\circ}$ (black)-all azimuths (purple). 
velocity is better than an inversion, which converges only to smooth velocities.

In light of the radiation patterns analysis presented above, a tradeoff between different parameters always exists, and inverting for even the simplest acoustic model representation of an orthorhombic medium is a dilemma. We think that an orthorhombic model inversion should be tackled through a hierarchical approach, in which we start by recovering the parameters that have the most influence on the data. The new parameterization style introduced in the next section is particularly convenient for such strategy.

\section{A NEW PARAMETERIZATION STYLE FOR ORTHORHOMBIC MEDIA}

A practical inversion strategy which starts updating the parameters that have the most influence on the recorded data, should benefit from representing the model by one velocity and dimensionless parameters. In this case, the wave sensitivity to the long and short wavelength variation of the velocity model is angular independent, and the dimensionless parameters provide the angular dependence. However, the dependence of waves propagating in an orthorhombic medium (contrary to the VTI case) varies not only with scattering angles but also with azimuths. This may result in a challenging data selection (filtering) step when inverting for the anisotropy param- eters, especially if the orientation of the symmetry planes (fractures orientation) is not accurately known, or the subsurface structure is made of different orthorhombic layers with different orientation of symmetry planes. Moreover, the conventional orthorhombic notation style stems from an elastic representation (Tsvankin, 1997), and thus has an inherent constraint of describing the model around the vertical velocity. This constraint reduces the number of possible acoustic model representation, and appeal to an alternative parameterization style.

\section{Definition of the deviation parameters}

Using the relations between the NMO velocities, the horizontal and the vertical velocities shown below

$$
\begin{array}{ll}
v_{h 1}^{2}=v_{v}^{2}\left(1+2 \epsilon_{1}\right), & v_{h 2}^{2}=v_{v}^{2}\left(1+2 \epsilon_{2}\right), \\
v_{n 1}^{2}=v_{v}^{2}\left(1+2 \delta_{1}\right), & v_{n 2}^{2}=v_{v}^{2}\left(1+2 \delta_{2}\right),
\end{array}
$$

we define the parameters $\epsilon_{d}, \delta_{d}$, and $\eta_{d}$ :

$$
\epsilon_{d} \equiv 0.5\left(\frac{v_{h 2}^{2}}{v_{h 1}^{2}}-1\right) \text {, }
$$

a)

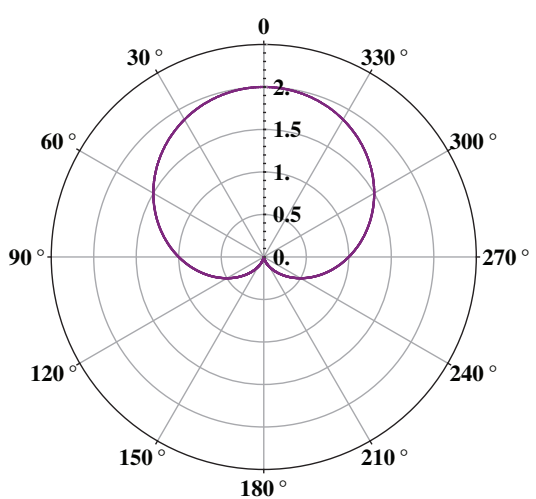

d)

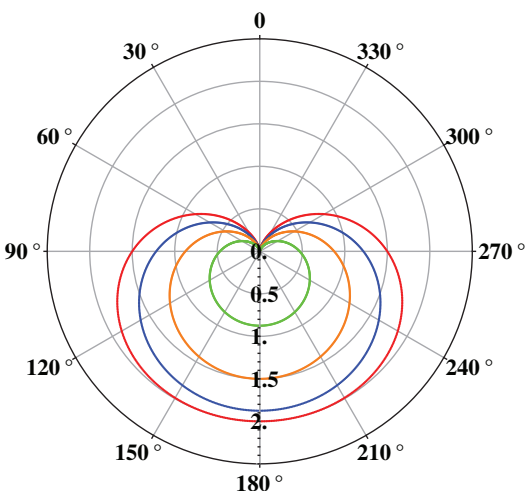

b)

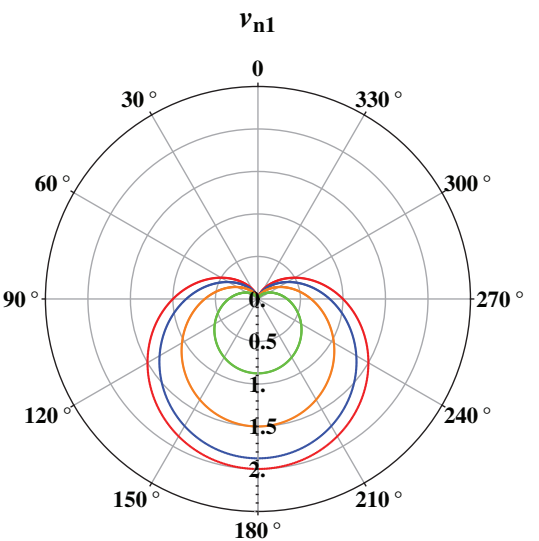

e)

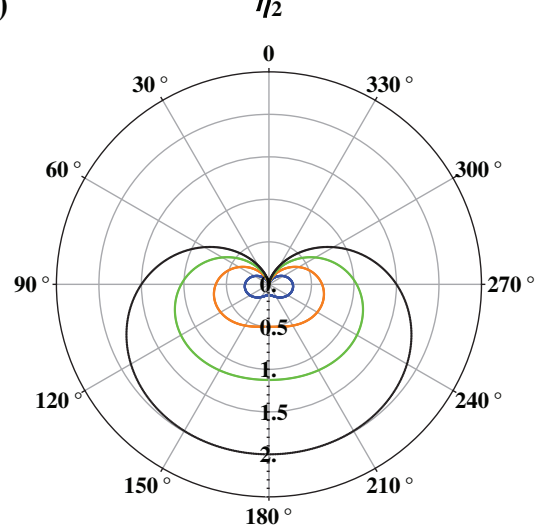

c)

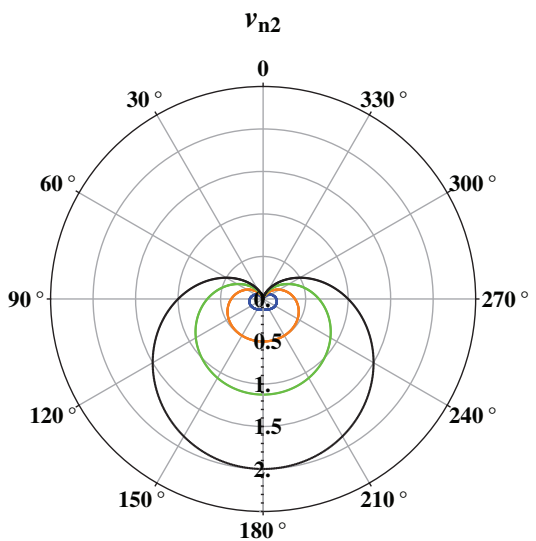

f)

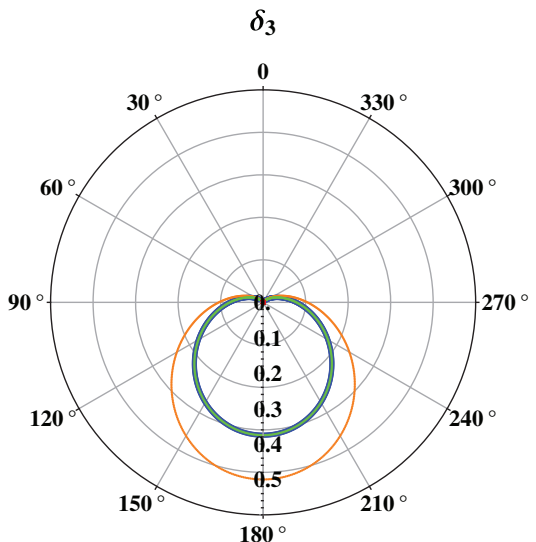

Figure 3. Radiation patterns for the parameterization with $\left(v_{v}, v_{n 1}, v_{n 2}, \eta_{1}, \eta_{2}, \delta_{3}\right)$ as a function of the scattering angle for different azimuth angles $\phi$ : $0^{\circ}$ (red), $30^{\circ}$ (blue), $45^{\circ}$ (orange), $60^{\circ}$ (green), $90^{\circ}$ (black)-all azimuths (purple). 


$$
\begin{gathered}
\delta_{d} \equiv 0.5\left(\frac{v_{n 2}^{2}}{v_{n 1}^{2}}-1\right), \\
\eta_{d} \equiv 0.5\left(\frac{v_{h 2}^{2} v_{n 1}^{2}}{v_{h 1}^{2} v_{n 2}^{2}}-1\right) .
\end{gathered}
$$

The parameters $\epsilon_{d}, \delta_{d}$, and $\eta_{d}$ are dimensionless and measure the deviation between the vertical planes in orthorhombic anisotropy. Such deviations can be measured by other combinations of parameters, however, we find those to be convenient. They also have simple expressions in terms of Thomsen-Tsvankin parameters:

$$
\begin{gathered}
\epsilon_{d}=\frac{\epsilon_{2}-\epsilon_{1}}{1+2 \epsilon_{1}}, \\
\delta_{d}=\frac{\delta_{2}-\delta_{1}}{1+2 \delta_{1}}, \\
\eta_{d}=\frac{\eta_{2}-\eta_{1}}{1+2 \eta_{1}}=\frac{\epsilon_{d}-\delta_{d}}{1+2 \delta_{d}} .
\end{gathered}
$$

With the newly defined parameters, an acoustic orthorhombic medium can be described by six parameters: one can choose any three VTI parameters (e.g., $\left.v_{h_{1}}, \epsilon_{1}, \eta_{1}\right)$ related to one vertical sym- metry plane (here, the $[x, z]$-plane), two deviation parameters (e.g., $\left.\epsilon_{d}, \eta_{d}\right)$, and one parameter related to the horizontal symmetry plane (e.g., $\delta_{3}$ ). Using parameters that measure the anisotropy deviation of the orthorhombic model between one VTI vertical plane and the other has practical benefits in representing the model and inverting for the parameters. To illustrate the features of the new parameterization style, we study the scattering potential of parameters corresponding to two relevant orthorhombic parameterizations. Our choice of these parameterizations is driven by our experience in inverting for the VTI parameters, and the motivation to propose practical inversion strategies. The radiation patterns equations of these parameterizations are shown in Appendix A.

\section{An orthorhombic parameterization with $\left(v_{h 1}, \epsilon_{1}, \eta_{1}, \eta_{d}\right.$, $\left.\epsilon_{d}, \delta_{3}\right)$}

In the parameterization $\left(v_{h 1}, \epsilon_{1}, \eta_{1}, \eta_{d}, \epsilon_{d}, \delta_{3}\right), v_{h 1}, \epsilon_{1}$, and $\eta_{1}$ are the VTI parameters describing the $[x, z]$ vertical symmetry plane, $\epsilon_{d}$ and $\eta_{d}$ measure the deviation from that plane, and $\delta_{3}$ is the anisotropy parameter defined in the $[x, y]$ horizontal symmetry plane.

Figure 5 shows the radiation patterns for this parameterization as a function of the scattering angles for different azimuth angles. Interestingly, not only the scattering potential of $v_{h 1}$ is azimuthally independent (Figure 5a), this behavior also holds for the parameters a)

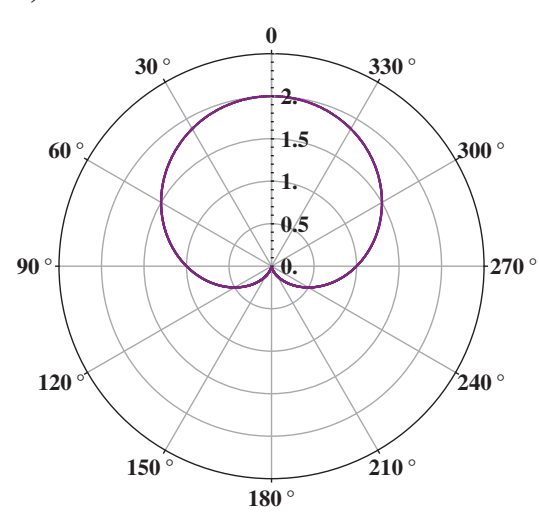

d)

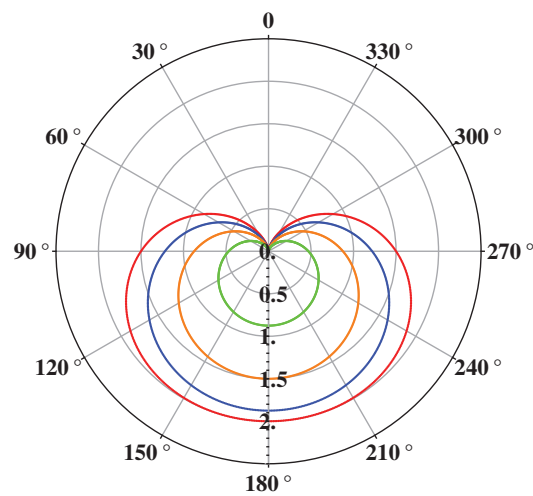

b)

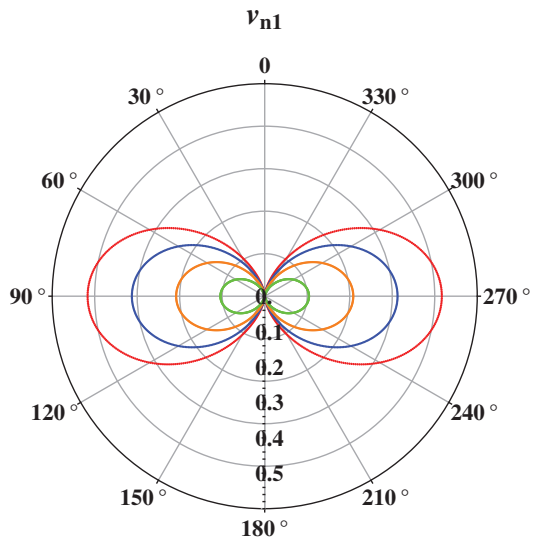

e)

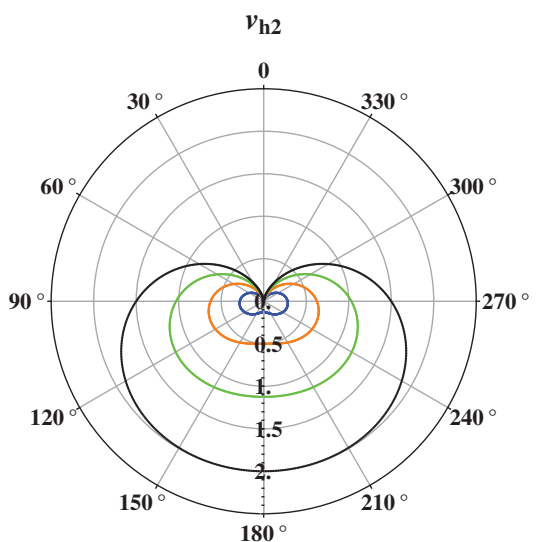

c)

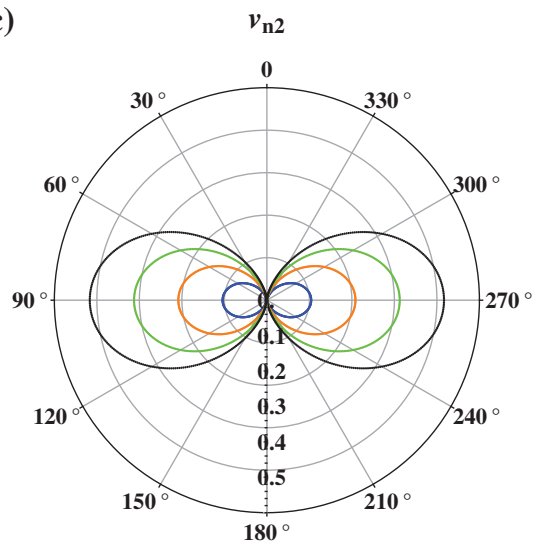

f)

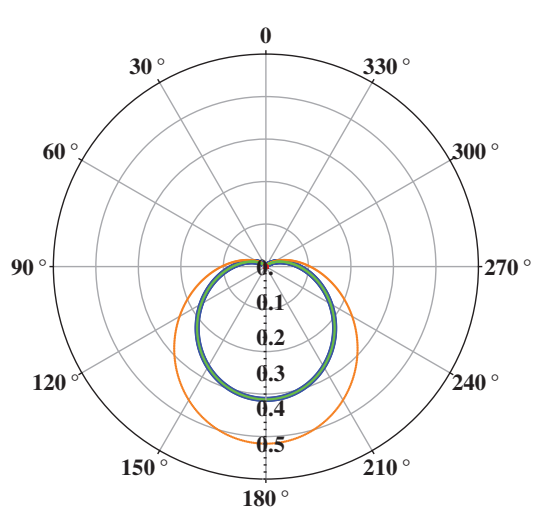

Figure 4. Radiation patterns for the parameterization with $\left(v_{v}, v_{n 1}, v_{n 2}, v_{h 1}, v_{h 2}, \delta_{3}\right)$ as a function of the scattering angle for different azimuth angles $\phi$ : $0^{\circ}$ (red), $30^{\circ}$ (blue), $45^{\circ}$ (orange), $60^{\circ}$ (green), $90^{\circ}$ (black)-all azimuths (purple). 
$\epsilon_{1}$ and $\eta_{1}$ (Figure $5 \mathrm{~b}$ and $5 \mathrm{c}$ ). Moreover, the scattering potential of $v_{h 1}$ is independent of the scattering angle (like in isotropic media), whereas $\epsilon_{1}$ and $\eta_{1}$ provide the scattering angular dependency. Specifically, $\epsilon_{1}$ is associated with small scattering angles, whereas $\eta_{1}$ has mainly influence on intermediate angles. On the other hand, Figure 5d-5f shows that the behavior of the deviation parameters $\epsilon_{d}, \eta_{d}$ and also the parameter $\delta_{3}$ is azimuthally dependent. The $\epsilon_{d}$ and $\eta_{d}$ affect mainly the data at $90^{\circ}$ azimuth, but they have distinct scattering angle influence. Note the weak scattering potential of $\eta_{1}$, $\eta_{d}$, and $\delta_{3}$, which suggests that these parameters produce less scattering than the other parameters. Based on these observations, we propose two approaches for inverting the orthorhombic model parameters.

The first approach consists of a multistage model inversion strategy. At the first stage, an isotropic inversion can be applied, in which diving waves are used to resolve the horizontal velocity. At the second stage, one should focus on the two VTI parameters: $\epsilon_{1}$ can help better fit the amplitudes at small scattering angles due to the acoustic assumption, and $\eta_{1}$ can be resolvable from intermediate scattering angles. At the last stage (as the VTI parameters provide the best possible fit for such a model), the three remaining parameters could be updated along with the VTI ones. The advantage of this strategy lies in mitigating the complexity of a highly nonlinear multiparameter inversion at the first stage of the inversion process, and it stems from the azimuthal independency of the scattering potential of the VTI parameters.
The weak scattering potential of the parameters $\eta_{1}, \eta_{d}$, and $\delta_{3}$ suggests another inversion strategy in which, one can simultaneously invert for $v_{h 1}, \epsilon_{1}$, and $\epsilon_{d}$, keeping the parameters $\eta_{1}, \eta_{d}$, and $\delta_{3}$ constant. The scattering influence of $\eta$ on surface seismic data for the VTI parameterization $\left(v_{h}, \eta, \epsilon\right)$ is described by Alkhalifah (2016), who recommends building the $\eta$ model from a tomographic inversion. In this parameterization choice, $v_{h 1}$ is uniquely constrained by large scattering angles and small azimuths, $\epsilon_{1}$ can absorb the amplitude mismatch in reflections due to the elastic nature of the data, and $\epsilon_{d}$ is constrained from diving waves for a source-to-receiver azimuth close to $90^{\circ}$. The second strategy is simpler, and it takes advantage of our experience with the VTI model inversion.

\section{An orthorhombic parameterization with $\left(v_{n 1}, \eta_{1}, \delta_{1}\right.$, $\left.\epsilon_{d}, \delta_{d}, \delta_{3}\right)$}

The radiation patterns corresponding to the parameterization $\left(v_{n 1}, \eta_{1}, \delta_{1}, \epsilon_{d}, \delta_{d}, \delta_{3}\right)$ are shown in Figure 6. Note the azimuthal independency of scattering of the VTI parameters $v_{n 1}, \eta_{1}$, and $\delta_{1}$ (Figure 6a-6c). This feature holds for any combination of VTI parameters. Moreover, we use the deviation parameter $\epsilon_{d}$, instead of $\eta_{d}$ (one might think about a symmetry, in which a parameter and the deviation from it are both present in the chosen parameterization) because a combination of $\epsilon_{d}$ and $\delta_{d}$ influences the data at distinct scattering angles, as shown in Figure $6 \mathrm{~d}$ and $6 \mathrm{e}$, whereas a a)

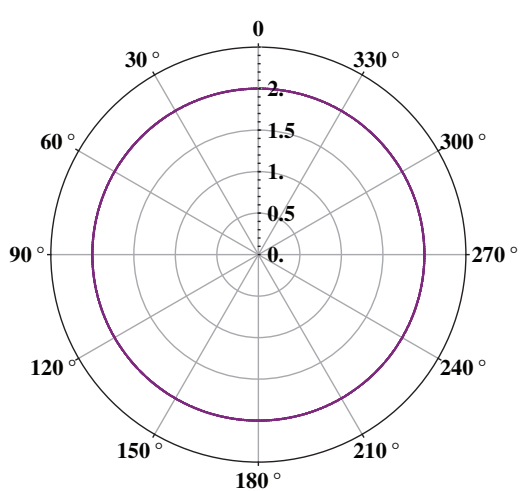

d)

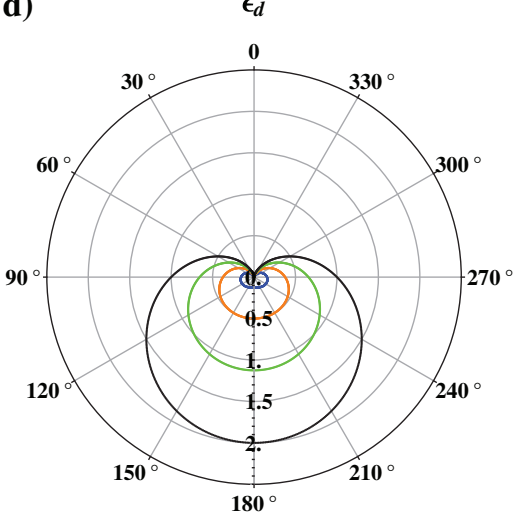

b)

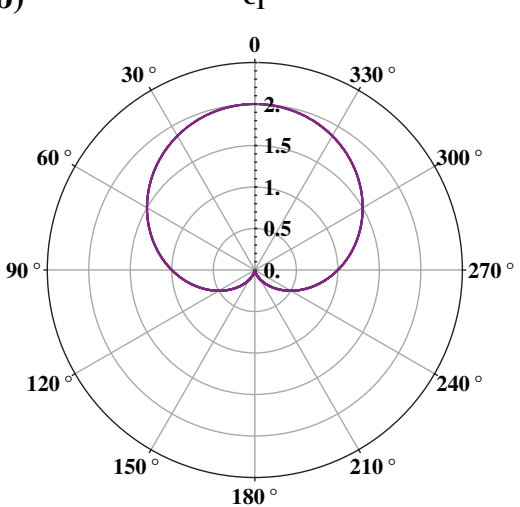

e)

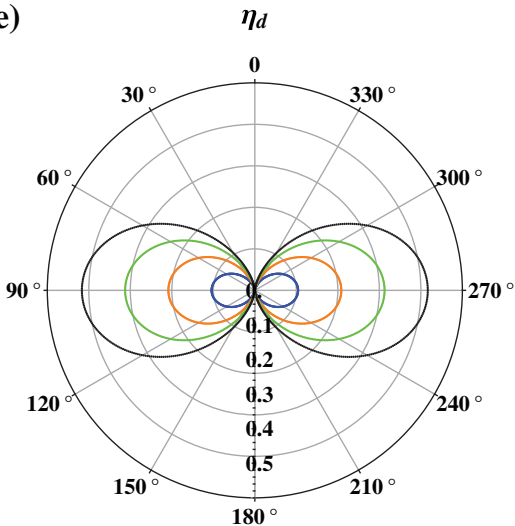

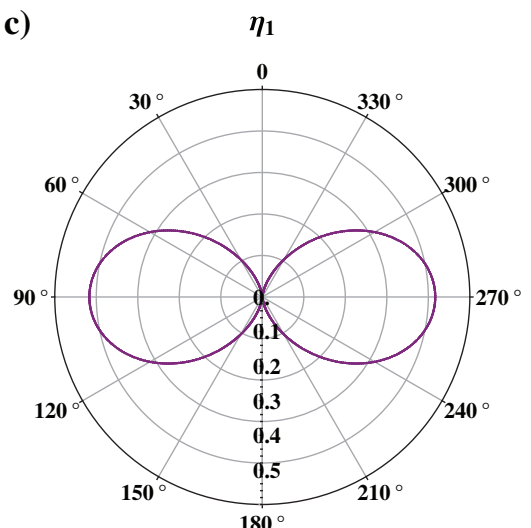

c)

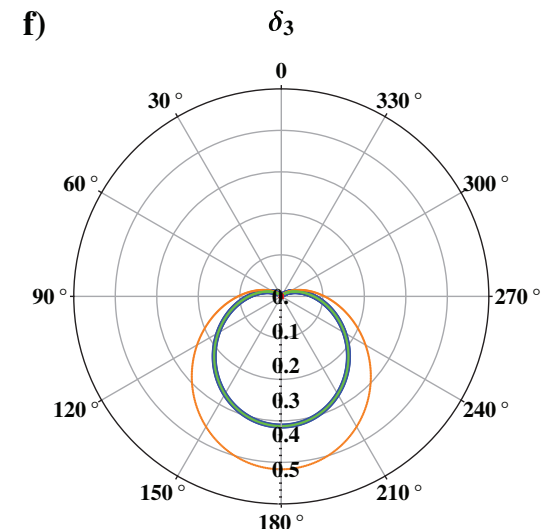

Figure 5. Radiation patterns for the parameterization with $\left(v_{h 1}, \epsilon_{1}, \eta_{1}, \epsilon_{d}, \eta_{d}, \delta_{3}\right)$ as a function of the scattering angle for different azimuth angles $\phi$ : $0^{\circ}$ (red), $30^{\circ}$ (blue), $45^{\circ}$ (orange), $60^{\circ}$ (green), $90^{\circ}$ (black)-all azimuths (purple). 
combination of $\eta_{d}$ and $\delta_{d}$ makes both parameters effecting the data at large offsets. This flexibility is convenient, and follows from equation 23. Due to the trade-off between $v_{n 1}$ and $\eta_{1}$ at large scattering angles and different azimuths, $v_{n 1}$ is not well-resolvable from diving waves. Unless the NMO velocity is constrained from reflection tomography, FWI should perform better with the parameterization with $v_{h 1}$.

We emphasize that the analysis above stems from our experience in analyzing the radiation patterns of the VTI parameters that we observe here (for the VTI parameters of the orthorhombic model).

\section{SENSITIVITY OF THE DATA TO TRAVELTIME PERTURBATION}

Because the data are sensitive to the traveltimes and the corresponding geometric amplitudes, we can gain insights into the behavior of FWI by studying the sensitivity of the data to traveltime variation caused by perturbation in the model parameters. We will study this sensitivity for two orthorhombic parameterizations, based on one velocity and five dimensionless parameters.

Traveltimes in anisotropic media can be obtained by solving the eikonal equation (Vidale, 1990; Van Trier and Symes, 1991). For orthorhombic media, the eikonal equation takes the form shown below (Alkhalifah, 2003):

$$
\begin{aligned}
& A_{i}\left(\frac{\partial \tau}{\partial x}\right)^{2}+B_{i}\left(\frac{\partial \tau}{\partial y}\right)^{2}+C_{i}\left(\frac{\partial \tau}{\partial z}\right)^{2} \\
& \quad+D_{i}\left(\frac{\partial \tau}{\partial x}\right)^{2}\left(\frac{\partial \tau}{\partial y}\right)^{2}+E_{i}\left(\frac{\partial \tau}{\partial x}\right)^{2}\left(\frac{\partial \tau}{\partial z}\right)^{2} \\
& \quad+F_{i}\left(\frac{\partial \tau}{\partial y}\right)^{2}\left(\frac{\partial \tau}{\partial z}\right)^{2}+G_{i}\left(\frac{\partial \tau}{\partial x}\right)^{2}\left(\frac{\partial \tau}{\partial y}\right)^{2}\left(\frac{\partial \tau}{\partial z}\right)^{2}=1,
\end{aligned}
$$

where $\tau(x, y, z)$ is the traveltime at a grid point with coordinates $(x, y, z)$. The coefficients $A_{i}, B_{i}, C_{i}, D_{i}, E_{i}, F_{i}$, and $G_{i}$ depend on the considered parameterization (see Appendix B).

Using perturbation theory, we expand the traveltime solution of the eikonal equation 24 in a Taylor's series expansion as follows:

$$
\tau(x, y, z) \approx \tau_{0}(x, y, z)+\sum_{i=1}^{5} \tau_{\alpha_{i}}(x, y, x) \alpha_{i},
$$

where $\tau_{0}$ is the isotropic background traveltime and the coefficients $\tau_{\alpha_{i}}(i=1, \ldots, 5)$ are perturbations in traveltimes induced by perturbation of the dimensionless parameters $\alpha_{i}$.

For each of the parameterization $\left(v_{h 1}, \epsilon_{1}, \eta_{1}, \eta_{d}, \epsilon_{d}, \delta_{3}\right)$ and $\left(v_{n 1}\right.$, $\left.\eta_{1}, \delta_{1}, \delta_{d}, \epsilon_{d}, \delta_{3}\right)$, we insert the trial solution 25 into the eikonal equation 24 , and we solve for the coefficients $\tau_{\alpha_{i}}$ of the expansion.
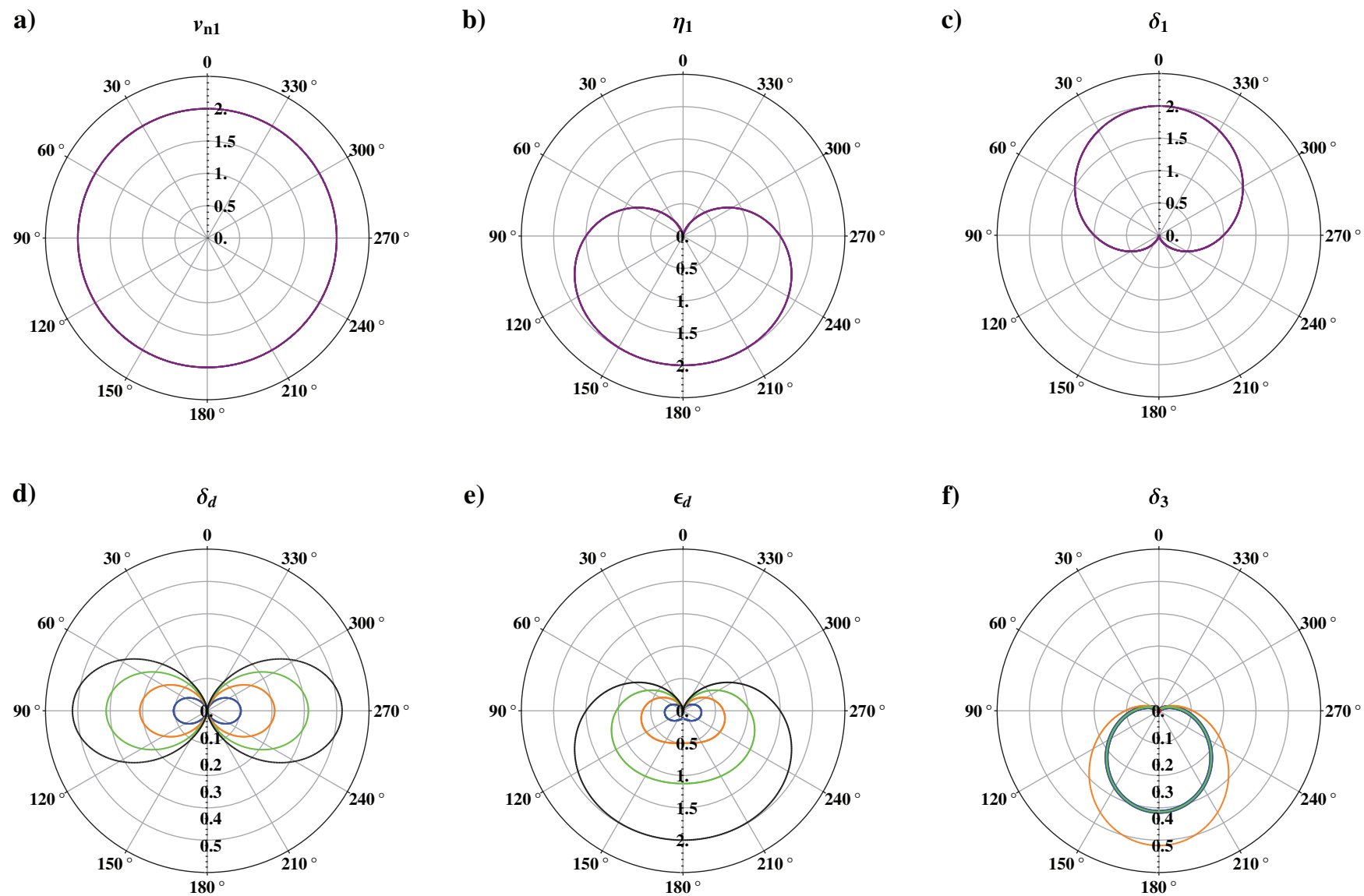

Figure 6. Radiation patterns for the parameterization with $\left(v_{n 1}, \eta_{1}, \delta_{1}, \delta_{d}, \epsilon_{d}, \delta_{3}\right)$ as a function of the scattering angle for different azimuth angles $\phi$ : $0^{\circ}$ (red), $30^{\circ}$ (blue), $45^{\circ}$ (orange), $60^{\circ}$ (green), $90^{\circ}$ (black)-all azimuths (purple). 
As shown in Appendix B, we obtain linear partial differential equations (in terms of $\tau_{\alpha_{i}}$ ) that have this form:

$$
\frac{\partial \tau_{0}}{\partial x} \frac{\partial \tau_{\alpha_{i}}}{\partial x}+\frac{\partial \tau_{0}}{\partial y} \frac{\partial \tau_{\alpha_{i}}}{\partial y}+\frac{\partial \tau_{0}}{\partial z} \frac{\partial \tau_{\alpha_{i}}}{\partial z}=f_{\alpha_{i}}, \quad i=\{1, \ldots, 5\},
$$

where $f_{\alpha_{i}}$ is the source function. We solve analytically equation 26 for the two considered parameterizations to analyze the influence of each parameter of the parameterization on the long wavelength components of the model.

\section{Traveltime perturbation for the parameterization with} $\left(v_{h 1}, \epsilon_{1}, \eta_{1}, \eta_{d}, \epsilon_{d}, \delta_{3}\right)$

By considering an isotropic background $\left(v_{h 1}=2 \mathrm{~km} / \mathrm{s}\right)$ and a source at $z=1 \mathrm{~km}$, we compute as shown in Figure 7, the analytical solutions of $\tau_{\epsilon_{1}}, \tau_{\eta_{1}}, \tau_{\eta_{d}}, \tau_{\epsilon_{d}}, \tau_{\delta_{3}}$, as a function of the source incident angles $\varphi_{x}=\operatorname{Tan}^{-1}(x / z)$ and $\varphi_{y}=\operatorname{Tan}^{-1}(y / z)$. Figure $7 \mathrm{a}$ showing the variation of $\tau_{\epsilon_{1}}$, reveals that $\epsilon_{1}$ has the largest influence at small incident angles $\varphi_{x}$ and $\varphi_{y}$ (small offsets) and has an azimuthally independent effect. This behavior is consistent with the radiation patterns of $\epsilon_{1}$ in the parameterization $\left(v_{h 1}, \epsilon_{1}, \eta_{1}, \eta_{d}\right.$, $\epsilon_{d}, \delta_{3}$ ), shown in Figure 5b. Similar azimuthally independent behavior is observed for $\eta_{1}$, as shown in Figure $7 \mathrm{~b}$. In this case, $\eta_{1}$ has its largest influence on incident angles between $45^{\circ}$ and $60^{\circ}$ (scattering angle between $90^{\circ}$ and $120^{\circ}$ ), similar to its radiation patterns shown in Figure 5c. Furthermore, Figure $7 \mathrm{c}$ and $7 \mathrm{~d}$ shows that $\epsilon_{d}$ and $\eta_{d}$ influences are mainly near the $\varphi_{y}$ axis direction (corresponding to an azimuth $\phi=90^{\circ}$ ). In addition, $\epsilon_{d}$ affects large incident angle $\varphi_{y}$, whereas $\eta_{d}$ influences intermediate incident angle. One can observe similar behavior on the radiation patterns in Figure $5 \mathrm{~d}$ and $5 \mathrm{e}$. Finally, $\delta_{3}$ (Figure 7e) affects large incident angle and an azimuth $\phi=45^{\circ}$ away from the $x$-axis, which is similar to the behavior of its radiation pattern.

These traveltime perturbation maps not only reveal the angular influence of each parameter but also give us insights into the sensitivity (in terms of amplitude) of the data to each parameter. This amplitude behavior seems to be consistent with the scattering potential found in the radiation patterns.

\section{Traveltime perturbation for the parameterization with $\left(v_{n 1}, \eta_{1}, \delta_{1}, \delta_{d}, \epsilon_{d}, \delta_{3}\right)$}

By considering an isotropic background $\left(v_{n 1}=2 \mathrm{~km} / \mathrm{s}\right)$ and a source at $z=1 \mathrm{~km}$, we compute the traveltime sensitivity maps of the parameters $\eta_{1}, \delta_{1}, \delta_{d}, \epsilon_{d}, \delta_{3}$ as a function of incident angles (Figure 8). In this parameterization, as shown in Figure $8 \mathrm{a}$ and $8 \mathrm{~b}$, $\eta_{1}$ influences the data at large angles of incidence, whereas $\delta_{1}$ has the main influence on the small angles. The values $\tau_{\eta_{1}}$ and $\tau_{\delta_{1}}$ are azimuthally independent, which is consistent with their radiation patterns. Moreover, Figure $8 \mathrm{c}$ and $8 \mathrm{~d}$ shows that $\delta_{d}$ and $\epsilon_{d}$ mainly influence the $y$-offset direction. Specifically, $\delta_{d}$ has its largest sensitivity for incident angles between $45^{\circ}$ and $60^{\circ}$ (scattering angles

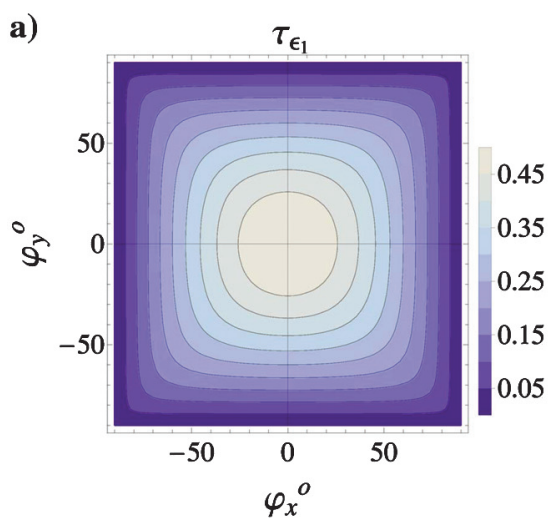

d)

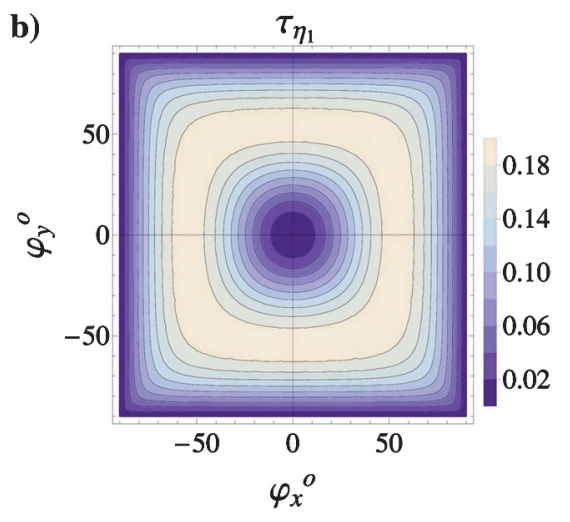

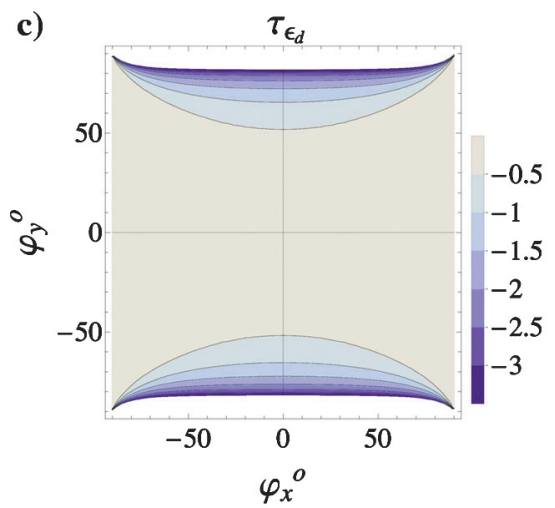

e)

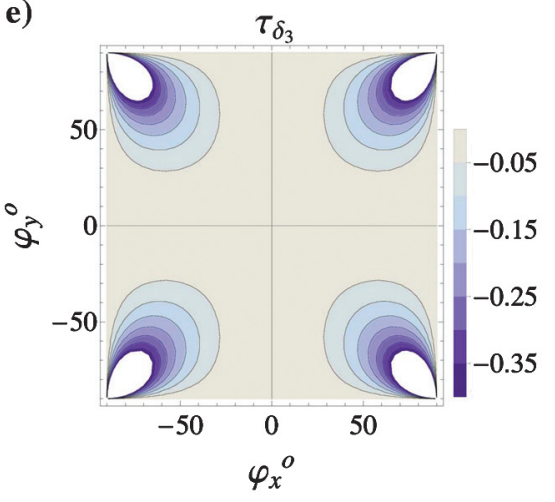

Figure 7. First-order traveltimes sensitivity as a function of incidence angles $\left(\varphi_{x}, \varphi_{y}\right)$ induced by perturbation in the orthorhombic model parameters (a) $\eta_{1}$, (b) $\epsilon_{1}$, (c) $\epsilon_{d}$, (d) $\eta_{d}$, and (e) $\delta_{3}$. The perturbation is from an isotropic background with $v_{h 1}=2 \mathrm{~km} / \mathrm{s}$, and the source is at $z=1 \mathrm{~km}$ depth. 
between $90^{\circ}$ and $120^{\circ}$ ), whereas $\epsilon_{d}$ has the maximum amplitude at large $\varphi_{y}$ incident angles. Finally, the behavior of $\delta_{3}$ (Figure 8e) in this parameterization is similar to the one observed in the previous case.

The consistency between the radiation patterns and the sensitivity traveltime maps allows us to validate the behavior of the new parameterization style. The sensitivity traveltime maps are high-frequency asymptotic approximation of the transmission part of the Born-based scattering potential. That part is expected to suffer from the depth ambiguity associated with surface seismic data. Contrary to the radiation patterns, the traveltime maps give better descriptions of the amplitude influence of each parameter, and as a consequence the proper weight that could be applied (Hessian) to balance such differences. In a nutshell, understanding fully the scattering amplitude and angular influence of each parameter in the chosen parameterization is one important ingredient for a successful multiparameter inversion.

\section{DISCUSSION}

Using Thomsen-Tsvankin notation style, we have limited parameterization choices for an orthorhombic medium, especially if the model is described by one velocity and five dimensionless parameters. On the other hand, the deviation parameters allow more versatile parameterization choice, and they offer more practical strategies to tackle an inversion problem. These strategies benefit from the azimuthal independency of the VTI parameters, and the different angular influence of the remaining parameters. As a consequence, we take advantage of our experience in dealing with isotropic and VTI inversions.

An orthorhombic medium describes one or two parallel vertical fracture sets in a VTI or an isotropic host rock. The definitions of deviation parameters representing the variation from a background model suggest that information about the fracture properties (fracture weaknesses) and attributes (crack density and fluid content) is related to the deviation parameters. Elucidating these dependencies and studying which properties could be reliably constrained from surface seismic data is essential for high-resolution reservoir characterization.

The traveltime maps describe the sensitivity nature of the data to the transmission part of the wavefield. Such transmission behavior is evident in the Born-based radiation pattern at one point $\left(180^{\circ}\right.$ scattering angle). The azimuthal behavior of this point coincides with the traveltime map results.

The study here is based on the Born approximation, and thus, adheres to the limitations associated with this approximation: small perturbations and low frequencies with respect to the scattering size. However, because the Born approximation provides us with the linear first-order component of the inversion update, given by the gradient, our conclusions here with respect to the scattering potential of the different parameterizations reveal the inherent trade-off we might face in inverting for these parameters. Using the Gauss Newton preconditioner in FWI may help to properly scale the update energy in the case of trade-off, which scale is based on the linear a)

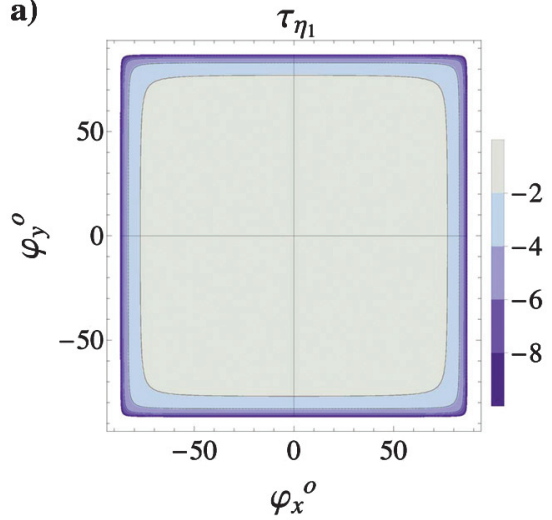

b)

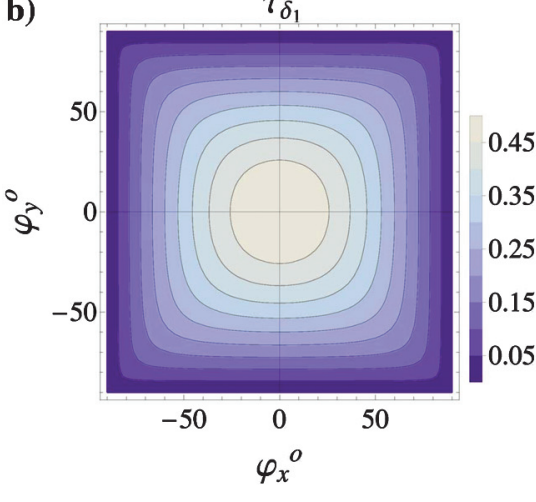

c)

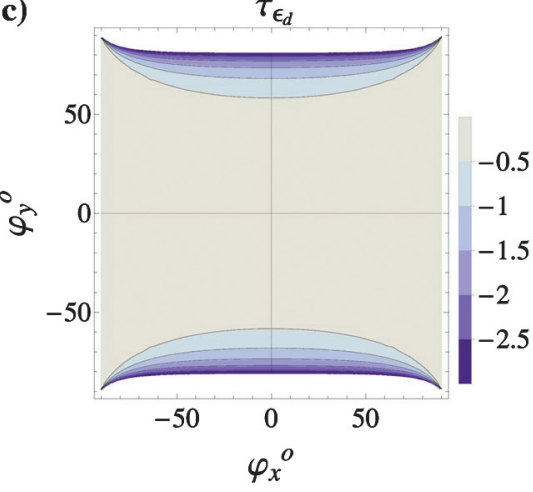

d)

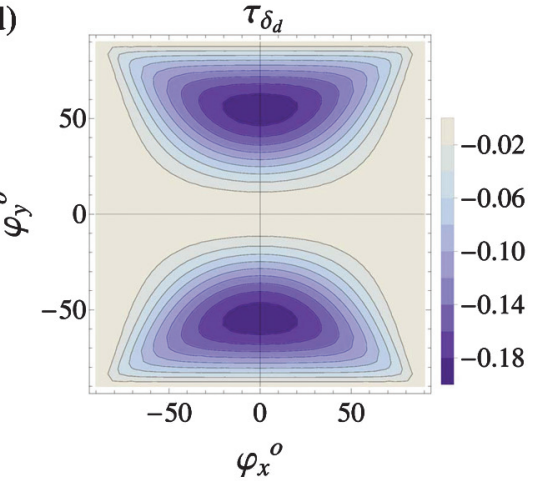

e)

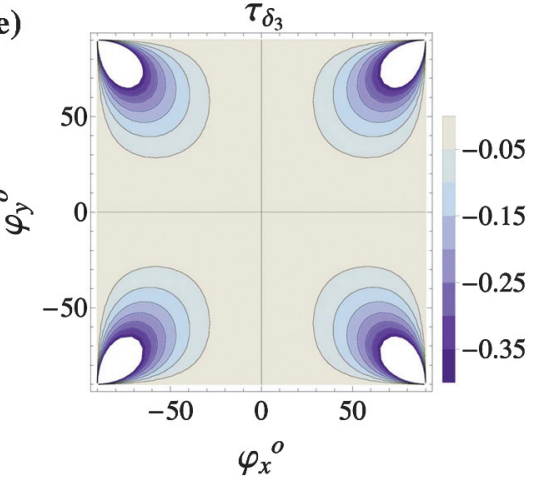

Figure 8. First-order traveltimes sensitivity as a function of incidence angles $\left(\varphi_{x}, \varphi_{y}\right)$ induced by perturbation in the orthorhombic model parameters (a) $\eta_{1}$, (b) $\delta_{1}$, (c) $\delta_{d}$, (d) $\epsilon_{d}$, and (e) $\delta_{3}$. The perturbation is from an isotropic background with $v_{n 1}=2 \mathrm{~km} / \mathrm{s}$, and the source is at $z=1 \mathrm{~km}$ depth. 
sensitivity kernel used to build the radiation pattern. This analysis may also reveal something not addressed by Gauss Newton preconditioner and that is a strategy for the inversion. Based on our acquisition setup for an orthorhombic inversion and the data sensitivity to the scattering of various parameters, we can exclude from the inversion parameters that have low influence on data at all or some azimuths, and also develop a multistage approach that ties inversion of particular data parts to particular parameters. With the newly introduced azimuth deviation parameters, we have such an opportunity knowing that we can start with an inversion of the isotropic parameter (velocity), followed by the VTI ones, and finally will all the azimuths the deviation parameters.

\section{CONCLUSIONS}

Orthorhombic model inversion is a challenge because in addition to the high nonlinearity due to the complexity of the model, we face the prospect of trade-off with multiparameter representation. Analyzing the scattering potential of various parameterizations of the orthorhombic model allowed us to suggest a parameter set that keeps the radiation patterns stationary as we move from isotropic for the (horizontal) velocity to a function of all angles for the VTI parameters $(\epsilon$ and $\eta$ ), and finally as a function of azimuth for the azimuth deviation parameters. We studied the radiation patterns of the conventional and a new parameterization style for orthorhombic media. The behavior of two particular parameterizations based on the deviation parameters $\epsilon_{d}, \eta_{d}$, and $\delta_{d}$ were also studied with a traveltime sensitivity approach. The strength of the sensitivity of the various parameters with respect to our multiazimuth surface seismic P-wave data acquisition suggests that we may focus the inversion on three parameters instead of the six, specifically dropping $\eta_{1}, \eta_{d}$, and $\delta_{3}$. For these parameters, we still need accurate background models of them from, for example, NMO analysis or tomography. The inversion of only three parameters promises to reduce the trade-off.

\section{ACKNOWLEDGMENTS}

We would like to thank KAUST for financial support. We thank R. Djebbi and U. bin Waheed for many useful discussions. We are also grateful to the associate editor, A. Stovas and two anonymous reviewers for their critical and helpful review of the paper.

\section{APPENDIX A}

\section{RADIATION PATTERNS FOR DIFFERENT ORTHORHOMBIC PARAMETERIZATIONS}

The radiation patterns in equations 14 and 15 correspond to the perturbation in $v_{n 1}^{2}, v_{n 2}^{2}, v_{v}^{2}, \eta_{1}, \eta_{2}$, and $\chi^{2}$ (equation 4). To derive the radiation patterns for any parameterization and perturbation, one can write the scattered field as follows:

$$
P_{s}=\omega^{6} \int G_{s}\left(\mathbf{x}_{s}, \mathbf{x}, \omega\right) G_{r}\left(\mathbf{x}_{r}, \mathbf{x}, \omega\right) a_{i}(\mathbf{x}) \cdot r_{i}(\mathbf{x}) d x,
$$

where $a_{i}$ is the radiation pattern for the perturbation parameters $r_{i}$. Using the relations between the parameters and the perturbations, we obtain the radiation patterns for the different parameterizations shown in this paper. For instance, from equations 1 , one can obtain the following relations (by assuming an isotropic background):

$$
\begin{aligned}
& r_{v_{n 1}^{2}}=r_{v_{v}^{2}}+2 r_{\delta_{1}}, \quad r_{v_{n 2}^{2}}=r_{v_{v}^{2}}+2 r_{\delta_{2}}, \\
& r_{v_{h 1}^{2}}=r_{v_{n 1}^{2}}+2 r_{\eta_{1}}, \quad r_{v_{h 2}^{2}}=r_{v_{n 2}^{2}}+2 r_{\eta_{2}}, \\
& r_{\eta_{1}}=r_{\epsilon_{1}}-r_{\delta_{1}}, \quad r_{\eta_{2}}=r_{\epsilon_{2}}-r_{\delta_{2}} .
\end{aligned}
$$

Moreover, because $\chi^{2}=1+2 \delta_{3}$, the perturbation $r_{\delta_{3}}$ is related to $r_{\chi^{2}}$ through this relation

$$
r_{\chi^{2}}=2 r_{\delta_{3}} .
$$

Using the reference radiation patterns $\left(a_{0}, r_{0}\right)$ given in equation 15 and the relations between the perturbations (equations A-2 and A3 ), we obtain the radiation patterns for the parameterization with one velocity and five dimensionless parameters $\left(v_{v}, \epsilon_{1}, \epsilon_{2}, \delta_{1}\right.$, $\left.\delta_{2}, \delta_{3}\right)$ as follows:

$$
\begin{aligned}
& a_{1}(\mathbf{x})=\left.\begin{array}{c}
2 \\
2 \sin ^{2} \theta \cos ^{2} \phi\left(1+\sin ^{2} \theta \sin ^{2} \phi+\cos ^{2} \theta\right) \\
2 \sin ^{2} \theta \sin ^{2} \phi\left(1-\sin ^{2} \theta \cos ^{2} \phi+\cos ^{2} \theta\right) \\
-2 \cos ^{2} \theta \sin ^{2} \theta \cos ^{2} \phi \\
-2 \cos ^{2} \theta \sin ^{2} \theta \sin ^{2} \phi \\
2 \sin ^{4} \theta \cos ^{2} \phi \sin ^{2} \phi
\end{array}\right), \\
& r_{1}(\mathbf{x})=\left(\begin{array}{c}
r_{v_{v}} \\
r_{\epsilon_{1}} \\
r_{\epsilon_{2}} \\
r_{\delta_{1}} \\
r_{\delta_{2}} \\
r_{\delta_{3}}
\end{array}\right),
\end{aligned}
$$

where $\theta$ and $\phi$ are the incident and azimuth angles, respectively. The radiation patterns for the parameterization with three velocities and three dimensionless parameters $\left(v_{n 1}, v_{n 2}, v_{v}, \eta_{1}, \eta_{2}, \delta_{3}\right)$ are given below:

$$
\begin{aligned}
a_{2}(\mathbf{x})= & \left(\begin{array}{c}
2 \sin ^{2} \theta \cos ^{2} \phi\left(1+\sin ^{2} \theta \sin ^{2} \phi\right) \\
2 \sin ^{2} \theta \sin ^{2} \phi\left(1-\sin ^{2} \theta \cos ^{2} \phi\right) \\
-2 \cos ^{2} \theta \\
2 \sin ^{2} \theta \cos ^{2} \phi\left(1+\sin ^{2} \theta \sin ^{2} \phi+\cos ^{2} \theta\right) \\
2 \sin ^{2} \theta \sin ^{2} \phi\left(1-\sin ^{2} \theta \cos ^{2} \phi+\cos ^{2} \theta\right) \\
2 \sin ^{4} \theta \cos ^{2} \phi \sin ^{2} \phi
\end{array}\right), \\
r_{2}(\mathbf{x})= & \left(\begin{array}{l}
r_{v_{n 1}} \\
r_{v_{n 2}} \\
r_{v_{v}} \\
r_{\eta_{1}} \\
r_{\eta_{2}} \\
r_{\delta_{3}}
\end{array}\right) .
\end{aligned}
$$

For the parameterization with five velocities and one dimensionless parameter $\left(v_{v}, v_{n 1}, v_{n 2}, v_{h 1}, v_{h 2}, \delta_{3}\right)$, the radiation patterns result into the following relations: 


$$
\begin{aligned}
a_{3}(\mathbf{x})=\left(\begin{array}{c}
-2 \cos ^{2} \theta \\
-2 \cos ^{2} \theta \sin ^{2} \theta \cos ^{2} \phi \\
-2 \cos ^{2} \theta \sin ^{2} \theta \sin ^{2} \phi \\
2 \sin ^{2} \theta \cos ^{2} \phi\left(1+\sin ^{2} \theta \sin ^{2} \phi+\cos ^{2} \theta\right) \\
2 \sin ^{2} \theta \sin ^{2} \phi\left(1-\sin ^{2} \theta \cos ^{2} \phi+\cos ^{2} \theta\right) \\
2 \sin ^{4} \theta \cos ^{2} \phi \sin ^{2} \phi
\end{array}\right), \\
r_{3}(\mathbf{x})=\left(\begin{array}{c}
r_{v_{v}} \\
r_{v_{n 1}} \\
r_{v_{n 2}} \\
r_{v_{h 1}} \\
r_{v_{h 2}} \\
r_{\delta_{3}}
\end{array}\right) .
\end{aligned}
$$

Using equations $18-23$, we obtain relations between the deviation anisotropy parameters and their perturbations, and we use them to compute the radiation patterns for the parameterization $\left(v_{h 1}, \epsilon_{1}, \eta_{1}\right.$, $\epsilon_{d}, \eta_{d}, \delta_{3}$ ), as shown below:

$$
\begin{aligned}
& a_{4}(\mathbf{x})=\left(\begin{array}{c}
2 \\
2 \cos ^{2} \theta \sin ^{2} \theta \\
-2 \cos ^{2} \theta \\
2 \cos ^{2} \theta \sin ^{2} \theta \sin ^{2} \phi \\
2 \sin ^{2} \theta \sin ^{2} \phi\left(1-\sin ^{2} \theta \cos ^{2} \phi\right) \\
2 \sin ^{4} \theta \cos ^{2} \phi \sin ^{2} \phi
\end{array}\right), \\
& r_{4}(\mathbf{x})=\left(\begin{array}{c}
r_{v_{h 1}} \\
r_{\eta_{1}} \\
r_{\epsilon_{1}} \\
r_{\eta_{d}} \\
r_{\epsilon_{d}} \\
r_{\delta_{3}}
\end{array}\right) .
\end{aligned}
$$

For the parameterization $\left(v_{n 1}, \eta_{1}, \delta_{1}, \delta_{d}, \epsilon_{d}, \delta_{3}\right)$, we obtain the following radiation patterns:

$$
\begin{aligned}
a_{5}(\mathbf{x}) & =\left(\begin{array}{c}
2 \\
(3+\cos 2 \theta) \sin ^{2} \theta \\
-2 \cos ^{2} \theta \\
-2 \cos ^{2} \theta \sin ^{2} \theta \sin ^{2} \phi \\
2 \sin ^{2} \theta \sin ^{2} \phi\left(1-\sin ^{2} \theta \cos ^{2} \phi+\cos ^{2} \theta\right) \\
2 \sin ^{4} \theta \cos ^{2} \phi \sin ^{2} \phi
\end{array}\right), \\
r_{5}(\mathbf{x})= & \left(\begin{array}{c}
r_{v_{n 1}} \\
r_{\eta_{1}} \\
r_{\delta_{1}} \\
r_{\delta_{d}} \\
r_{\epsilon_{d}} \\
r_{\delta_{3}}
\end{array}\right) .
\end{aligned}
$$

\section{APPENDIX B}

\section{TRAVELTIME PERTURBATION DIFFERENTIAL EQUATIONS}

The eikonal equation corresponding to orthorhombic anisotropy has the following form (Alkhalifah, 2003):

$$
\begin{aligned}
& A_{i}\left(\frac{\partial \tau}{\partial x}\right)^{2}+B_{i}\left(\frac{\partial \tau}{\partial y}\right)^{2}+C_{i}\left(\frac{\partial \tau}{\partial z}\right)^{2} \\
& \quad+D_{i}\left(\frac{\partial \tau}{\partial x}\right)^{2}\left(\frac{\partial \tau}{\partial y}\right)^{2}+E_{i}\left(\frac{\partial \tau}{\partial x}\right)^{2}\left(\frac{\partial \tau}{\partial z}\right)^{2} \\
& \quad+F_{i}\left(\frac{\partial \tau}{\partial y}\right)^{2}\left(\frac{\partial \tau}{\partial z}\right)^{2}+G_{i}\left(\frac{\partial \tau}{\partial x}\right)^{2}\left(\frac{\partial \tau}{\partial y}\right)^{2}\left(\frac{\partial \tau}{\partial z}\right)^{2}=1,
\end{aligned}
$$

where $\tau(x, y, z)$ is the traveltime at a grid point with coordinates $(x, y, z)$. The coefficients $A_{i}, B_{i}, C_{i}, D_{i}, E_{i}, F_{i}$, and $G_{i}$ depend on the considered parameterization. Using Taylor's series, we expand the solution of the eikonal equation B-1 in the following form:

$$
\tau(x, y, z) \approx \tau_{0}(x, y, z)+\sum_{i=1}^{5} \tau_{\alpha_{i}}(x, y, x) \alpha_{i},
$$

where $\tau_{0}$ is the background traveltime, and the coefficients $\tau_{\alpha_{i}}$ correspond to the five dimensionless parameters considered in the acoustic orthorhombic parameterization. In the following, we derive the coefficients of the traveltime expansion B-2 for two parameterizations based on the deviation parameters.

\section{Parameterization with $\left(v_{h 1}, \epsilon_{1}, \eta_{1}, \eta_{d}, \epsilon_{d}, \delta_{3}\right)$}

For a parameterization with $\left(v_{h 1}, \epsilon_{1}, \eta_{1}, \eta_{d}, \epsilon_{d}, \delta_{3}\right)$, the coefficients of the eikonal equation B-1 are shown below:

$$
\begin{aligned}
A_{1}= & v_{h 1}^{2}, \quad B_{1}=v_{h 1}^{2}\left(1+2 \epsilon_{d}\right), \quad C_{1}=\frac{v_{h 1}^{2}}{1+2 \epsilon_{1}}, \\
D_{1}= & v_{h 1}^{4}\left(\left(1+2 \delta_{3}\right)-\left(1+2 \epsilon_{d}\right)\right), \\
E_{1}= & -\frac{2 \eta_{1} v_{h 1}^{4}}{\left(1+2 \eta_{1}\right)\left(1+2 \epsilon_{1}\right)}, \\
F_{1}= & -\frac{v_{h 1}^{4}\left(1+2 \epsilon_{d}\right)\left(\left(1+2 \eta_{d}\right)\left(1+2 \eta_{1}\right)-1\right)}{\left(1+2 \eta_{d}\right)\left(1+2 \eta_{1}\right)\left(1+2 \epsilon_{1}\right)}, \\
G_{1}= & -\frac{v_{h 1}^{6}}{\left(1+2 \epsilon_{1}\right)\left(1+2 \eta_{1}\right)} \\
& \times\left(\left(1+2 \eta_{1}\right)\left(1+2 \delta_{3}\right)-2 \sqrt{\frac{\left(1+2 \epsilon_{d}\right)\left(1+2 \delta_{3}\right)}{1+2 \eta_{d}}}\right. \\
& \left.+\left(1+2 \epsilon_{d}\right)\left(-2 \eta_{1}+\frac{1}{1+2 \eta_{d}}\right)\right) .
\end{aligned}
$$

We insert the trial solution B-2 (using relation B-3) into the eikonal equation B-1 to derive expressions for $\tau_{0}$ and $\tau_{\alpha_{i}}\left(\alpha_{i}=\right.$ $\left.\epsilon_{1}, \eta_{1}, \eta_{d}, \epsilon_{d}, \delta_{3}\right)$. We obtain first a long formula, but by setting $\alpha_{i}=0$, one can retrieve the background traveltime $\tau_{0}$ in this form 


$$
\left(\frac{\partial \tau_{0}}{\partial x}\right)^{2}+\left(\frac{\partial \tau_{0}}{\partial y}\right)^{2}+\left(\frac{\partial \tau_{0}}{\partial z}\right)^{2}=\frac{1}{v_{h 1}^{2}}
$$

which is the eikonal equation for an isotropic medium. The remaining coefficients of the expansion satisfy first-order linear partial differential equations. After some manipulations, we obtain the following equations:

$$
\begin{gathered}
\frac{\partial \tau_{0}}{\partial x} \frac{\partial \tau_{\eta_{1}}}{\partial x}+\frac{\partial \tau_{0}}{\partial y} \frac{\partial \tau_{\eta_{1}}}{\partial y}+\frac{\partial \tau_{0}}{\partial z} \frac{\partial \tau_{\eta_{1}}}{\partial z} \\
=v_{h 1}^{2}\left(\frac{\partial \tau_{0}}{\partial z}\right)^{2}\left(\left(\frac{\partial \tau_{0}}{\partial x}\right)^{2}+\left(\frac{\partial \tau_{0}}{\partial y}\right)^{2}\right) \\
\frac{\partial \tau_{0}}{\partial x} \frac{\partial \tau_{\epsilon_{1}}}{\partial x}+\frac{\partial \tau_{0}}{\partial y} \frac{\partial \tau_{\epsilon_{1}}}{\partial y}+\frac{\partial \tau_{0}}{\partial z} \frac{\partial \tau_{\epsilon_{1}}}{\partial z}=\left(\frac{\partial \tau_{0}}{\partial z}\right)^{2} \\
\frac{\partial \tau_{0}}{\partial x} \frac{\partial \tau_{\eta_{d}}}{\partial x}+\frac{\partial \tau_{0}}{\partial y} \frac{\partial \tau_{\eta_{d}}}{\partial y}+\frac{\partial \tau_{0}}{\partial z} \frac{\partial \tau_{\eta_{d}}}{\partial z}=v_{h 1}^{2}\left(\frac{\partial \tau_{0}}{\partial z}\right)^{2}\left(\frac{\partial \tau_{0}}{\partial y}\right)^{2} \\
\frac{\partial \tau_{0}}{\partial x} \frac{\partial \tau_{\epsilon_{d}}}{\partial x}+\frac{\partial \tau_{0}}{\partial y} \frac{\partial \tau_{\epsilon_{d}}}{\partial y}+\frac{\partial \tau_{0}}{\partial z} \frac{\partial \tau_{\epsilon_{d}}}{\partial z} \\
\left.=\left(\frac{\partial \tau_{0}}{\partial y}\right)^{2}\left(\mathrm{~B}-\frac{\partial \tau_{0}}{\partial x}\right)^{2}-1\right)_{\delta_{3}}^{2}+\frac{\partial \tau_{0}}{\partial y} \frac{\partial \tau_{\delta_{3}}}{\partial y}+\frac{\partial \tau_{0}}{\partial z} \frac{\partial \tau_{\delta_{3}}}{\partial z}=-v_{h 1}^{2}\left(\frac{\partial \tau_{0}}{\partial x}\right)^{2}\left(\frac{\partial \tau_{0}}{\partial y}\right)^{2}
\end{gathered}
$$

We solve equations B-5 to B-9 analytically by considering a homogeneous isotropic background model. In this case, $\tau_{0}$ satisfies the following relation:

$$
\tau_{0}=\sqrt{\frac{x^{2}+y^{2}+z^{2}}{v_{h 1}^{2}}}
$$

where $x, y$, and $z$ are the coordinates of a Cartesian coordinate system. Using equation B-10, we solve equations B-5 to B-9, and obtain the following solutions:

$$
\begin{gathered}
\tau_{\eta_{1}}=\frac{\tau_{0} z^{2}\left(x^{2}+y^{2}\right)}{\left(x^{2}+y^{2}+z^{2}\right)^{2}}, \\
\tau_{\epsilon_{1}}=\frac{\tau_{0} z^{2}}{x^{2}+y^{2}+z^{2}}, \\
\tau_{\eta_{d}}=\frac{y^{2} z^{2} \tau_{0}}{\left(x^{2}+y^{2}+z^{2}\right)^{2}}, \\
\tau_{\epsilon_{d}}=-\frac{y^{2} \tau_{0}\left(y^{2}+z^{2}\right)}{\left(x^{2}+y^{2}+z^{2}\right)^{2}},
\end{gathered}
$$

$$
\tau_{\delta_{3}}=-\frac{x^{2} y^{2} \tau_{0}}{\left(x^{2}+y^{2}+z^{2}\right)^{2}}
$$

\section{Parameterization with $\left(v_{n 1}, \eta_{1}, \delta_{1}, \delta_{d}, \epsilon_{d}, \delta_{3}\right)$}

The coefficients of the eikonal equation B-1 for the parameterization $\left(v_{n 1}, \eta_{1}, \delta_{1}, \delta_{d}, \epsilon_{d}, \delta_{3}\right)$ are the following:

$$
\begin{aligned}
A_{2}= & v_{n 1}^{2}\left(1+2 \eta_{1}\right), \\
B_{2}= & v_{n 1}^{2}\left(1+2 \epsilon_{d}\right)\left(1+2 \eta_{1}\right), \\
C_{2}= & \frac{v_{n 1}^{2}}{1+2 \delta_{1}}, \\
D_{2}= & v_{n 1}^{4}\left(1+2 \eta_{1}\right)^{2}\left(\left(1+2 \delta_{3}\right)-\left(1+2 \epsilon_{d}\right)\right), \\
E_{2}= & -\frac{2 \eta_{1} v_{n 1}^{4}}{\left(1+2 \delta_{1}\right)}, \\
F_{2}= & -\frac{2 v_{n 1}^{4}\left(1+2 \delta_{d}\right)}{1+2 \delta_{1}}\left(\left(\frac{\epsilon_{d}-\delta_{d}}{1+2 \delta_{d}}\right)\left(1+2 \eta_{1}\right)+\eta_{1}\right), \\
G_{2}= & -\frac{v_{n 1}^{6}}{1+2 \delta_{1}}\left(\left(1+2 \eta_{1}\right)^{2}\left(1+2 \delta_{3}\right)\right. \\
& -2 \sqrt{\left(1+2 \delta_{3}\right)\left(1+2 \delta_{d}\right)} \\
& \left.+\left(1+2 \delta_{d}\right)\left(1-4 \eta_{1}\left(\frac{\epsilon_{d}-\delta_{d}}{1+2 \delta_{d}}\left(1+2 \eta_{1}\right)+\eta_{1}\right)\right)\right) .
\end{aligned}
$$

We insert the trial solution B-2 (using relation B-16) into the eikonal equation B-1 and derive the partial differential equation for $\tau_{0}$ and $\tau_{\alpha_{i}}\left(\alpha_{i}=\epsilon_{1}, \eta_{1}, \eta_{d}, \epsilon_{d}, \delta_{3}\right)$. First, by setting $\alpha_{i}=0$, one can retrieve the background traveltime $\tau_{0}$ in this form

$$
\left(\frac{\partial \tau_{0}}{\partial x}\right)^{2}+\left(\frac{\partial \tau_{0}}{\partial y}\right)^{2}+\left(\frac{\partial \tau_{0}}{\partial z}\right)^{2}=\frac{1}{v_{n 1}^{2}}
$$

The remaining coefficients satisfy linear partial differential equations as shown below:

$$
\begin{gathered}
\frac{\partial \tau_{0}}{\partial x} \frac{\partial \tau_{\eta_{1}}}{\partial x}+\frac{\partial \tau_{0}}{\partial y} \frac{\partial \tau_{\eta_{1}}}{\partial y}+\frac{\partial \tau_{0}}{\partial z} \frac{\partial \tau_{\eta_{1}}}{\partial z} \\
=v_{n 1}^{2}\left(\frac{\partial \tau_{0}}{\partial z}\right)^{2}\left(\left(\frac{\partial \tau_{0}}{\partial x}\right)^{2}+\left(\frac{\partial \tau_{0}}{\partial y}\right)^{2}\right. \\
\left.+2 v_{n 1}^{2}\left(\frac{\partial \tau_{0}}{\partial x}\right)^{2}\left(\frac{\partial \tau_{0}}{\partial y}\right)^{2}\right)-\left(\frac{\partial \tau_{0}}{\partial y}\right)^{2}-\left(\frac{\partial \tau_{0}}{\partial x}\right)^{2}, \\
\frac{\partial \tau_{0}}{\partial x} \frac{\partial \tau_{\delta_{1}}}{\partial x}+\frac{\partial \tau_{0}}{\partial y} \frac{\partial \tau_{\delta_{1}}}{\partial y}+\frac{\partial \tau_{0}}{\partial z} \frac{\partial \tau_{\delta_{1}}}{\partial z}=\left(\frac{\partial \tau_{0}}{\partial z}\right)^{2},
\end{gathered}
$$

$$
\frac{\partial \tau_{0}}{\partial x} \frac{\partial \tau_{\delta_{d}}}{\partial x}+\frac{\partial \tau_{0}}{\partial y} \frac{\partial \tau_{\delta_{d}}}{\partial y}+\frac{\partial \tau_{0}}{\partial z} \frac{\partial \tau_{\delta_{d}}}{\partial z}=-v_{n 1}^{2}\left(\frac{\partial \tau_{0}}{\partial z}\right)^{2}\left(\frac{\partial \tau_{0}}{\partial y}\right)^{2}
$$




$$
\begin{gathered}
\frac{\partial \tau_{0}}{\partial x} \frac{\partial \tau_{\epsilon_{d}}}{\partial x}+\frac{\partial \tau_{0}}{\partial y} \frac{\partial \tau_{\epsilon_{d}}}{\partial y}+\frac{\partial \tau_{0}}{\partial z} \frac{\partial \tau_{\epsilon_{d}}}{\partial z} \\
=\left(\frac{\partial \tau_{0}}{\partial y}\right)^{2}\left(v_{n 1}^{2}\left(\left(\frac{\partial \tau_{0}}{\partial x}\right)^{2}+\left(\frac{\partial \tau_{0}}{\partial z}\right)^{2}\right)-1\right), \\
\frac{\partial \tau_{0}}{\partial x} \frac{\partial \tau_{\delta_{3}}}{\partial x}+\frac{\partial \tau_{0}}{\partial y} \frac{\partial \tau_{\delta_{3}}}{\partial y}+\frac{\partial \tau_{0}}{\partial z} \frac{\partial \tau_{\delta_{3}}}{\partial z}=-v_{n 1}^{2}\left(\frac{\partial \tau_{0}}{\partial x}\right)^{2}\left(\frac{\partial \tau_{0}}{\partial y}\right)^{2} .
\end{gathered}
$$

We solve equations B-18 to B-22 analytically by considering a homogeneous isotropic background model. In this case, $\tau_{0}$ satisfies this relation

$$
\tau_{0}=\sqrt{\frac{x^{2}+y^{2}+z^{2}}{v_{n 1}^{2}}},
$$

and the remaining coefficients $\tau_{\eta_{1}}, \tau_{\delta_{1}}, \tau_{\delta_{d}}, \tau_{\epsilon_{d}}, \tau_{\delta_{3}}$ have the following solutions:

$$
\begin{gathered}
\tau_{\eta_{1}}=-\frac{\tau_{0}\left(x^{6}+3 x^{4} y^{2}+3 x^{2} y^{4}+y^{6}+x^{4} z^{2}+y^{4} z^{2}\right)}{\left(x^{2}+y^{2}+z^{2}\right)^{3}}, \\
\tau_{\delta_{1}}=\frac{\tau_{0} z^{2}}{x^{2}+y^{2}+z^{2}}, \\
\tau_{\delta_{d}}=-\frac{y^{2} z^{2} \tau_{0}}{\left(x^{2}+y^{2}+z^{2}\right)^{2}}, \\
\tau_{\epsilon_{d}}=-\frac{y^{4} \tau_{0}}{\left(x^{2}+y^{2}+z^{2}\right)^{2}}, \\
\tau_{\delta_{3}}=-\frac{x^{2} y^{2} \tau_{0}}{\left(x^{2}+y^{2}+z^{2}\right)^{2}} .
\end{gathered}
$$

\section{REFERENCES}

Alkhalifah, T., 2003, An acoustic wave equation for orthorhombic anisotropy: Geophysics, 68, 1169-1172, doi: 10.1190/1.1598109.

Alkhalifah, T., 2016, Research note: Insights into the data dependency on anisotropy: An inversion prospective: Geophysical Prospecting, 64, 505513, doi: 10.1111/1365-2478.12345.

Alkhalifah, T., and R. E. Plessix, 2014, A recipe for practical full-waveform inversion in anisotropic media: An analytical parameter resolution study: Geophysics, 79, no. 3, R91-R101, doi: 10.1190/geo2013-0366.1.

Barnes, C., M. Charara, and T. Tsuchiya, 2008, Feasibility study for an anisotropic full waveform inversion of cross-well seismic data: Geophysical Prospecting, 56, 897-906, doi: 10.1111/j.1365-2478.2008.00702.x.

Burridge, R., M. de Hoop, D. Miller, and C. Spencer, 1998, Multiparameter inversion in anisotropic elastic media: Geophysical Journal International, 134, 757-777, doi: 10.1046/j.1365-246x.1998.00590.x.

Cheng, X., K. Jiao, D. Sun, and D. Vigh, 2014, Multiparameter full-waveform inversion for acoustic VTI medium with surface seismic data: 76th Annual International Conference and Exhibition, EAGE, Extended Abstracts, We E106 04

Cohen, J. K., and N. Bleistein, 1977, An inverse method for determining small variations in propagation speed: SIAM Journal of Applied Mathematics, 32, 784-799, doi: 10.1137/0132066.

da Silva, N., A. Ratcliffe, G. Conroy, V. Vinje, and G. Body, 2014, Using full waveform inversion to update anisotropy — A North Sea case study: 76th
Annual International Conference and Exhibition, EAGE, Extended Abstracts, We E106 08

Djebbi, R., and T. Alkhalifah, 2014, Analysis of the multi-component pseudo-pure-mode qP-wave inversion in vertical transverse isotropic (VTI) media: 76th Annual International Conference and Exhibition, EAGE, Extended Abstracts, 394-399.

Forgues, E., and G. Lambaré, 1997, Parameterization study for acoustic and elastic ray + Born inversion: Journal of Seismic Exploration, 6, 253-278. Gholami, Y., R. Brossier, S. Operto, A. Ribodetti, and J. Virieux, 2013a Which parameterization is suitable for acoustic vertical transverse isotropic media full waveform inversion? Part 1: Sensitivity and tradeoff analysis: Geophysics, 78, no. 2, R81-R105, doi: 10.1190/ geo2012-0204.1.

Gholami, Y., R. Brossier, S. Operto, A. Ribodetti, and J. Virieux, 2013b, Which parameterization is suitable for acoustic vertical transverse isotropic media full waveform inversion? Part 2: Synthetic and real data case studies from Valhall: Geophysics, 78, no. 2, R107-R124, doi: 10.1190/ geo2012-0203.1.

Grechka, V., and I. Tsvankin, 1999, 3D moveout velocity analysis and parameter estimation for orthorhombic media: Geophysics, 64, 820837, doi: 10.1190/1.1444593.

Ibanez-Jacome, W., T. Alkhalifah, and U. Waheed, 2014, Effective orthorhombic anisotropic models for wavefield extrapolation: Geophysical Journal International, 198, 1653-1661, doi: 10.1093/gii/ggu229.

Kiyashchenko, D., B. Kashtan, and R.-E. Plessix, 2004, Anisotropic migration weight for amplitude-preserving migration and sensitivity analysis: Geophysical Journal International, 157, 753-763, doi: 10.1111/j .1365-246X.2004.02242.x.

Operto, S., A. Miniussi, R. Brossier, L. Combe, N. Haller, E. Kjos, L. Métivier, R. Milne, A. Ribodetti, Z. Song, J. Virieux, and Y. Zheng, 2015, Efficient 3D frequency-domain full-waveform inversion of ocean-bottom cable data - Application to Valhall in the visco-ac: 77th Annual International Conference and Exhibition, EAGE, Extended Abstracts, Th N104 04

Panning, M. P., Y. Capdeville, and B. A. Romanowicz, 2009, Seismic waveform modelling in a 3-D earth using the Born approximation: Potential shortcomings and a remedy: Geophysical Journal International, 177, 161178, doi: 10.1111/j.1365-246X.2008.04050.x.

Pratt, R. G., 1999, Seismic waveform inversion in the frequency domain. Part 1: Theory and verification in a physical scale model: Geophysics, 64, 888-901, doi: 10.1190/1.1444597.

Prieux, V., R. Brossier, Y. Gholami, S. Operto, J. Virieux, O. I. Barkved, and J. H. Kommedal, 2011, On the footprint of anisotropy on isotropic full waveform inversion: The Valhall case study: Geophysical Journal International, 187, 1495-1515, doi: 10.1111/j.1365-246X.2011.05209.x.

Prieux, V., R. Brossier, S. Operto, and J. Virieux, 2013, Multiparameter full waveform inversion of multicomponent ocean-bottom-cable data from the Valhall field. Part 1: Imaging compressional wave speed, density and attenuation: Geophysical Journal International, 194, 1640-1664, doi: 10 $.1093 / \mathrm{gji} / \mathrm{ggt} 177$.

Schoenberg, M., and K. Helbig, 1997, Orthorhombic media: Modeling elastic wave behavior in a vertically fractured earth: Geophysics, 62, 1954 1974, doi: 10.1190/1.1444297.

Stovas, A., 2015, Azimuthally dependent kinematic properties of orthorhombic media: Geophysics, 80, no. 6, C107-C122, doi: 10.1190/ geo2015-0288.1.

Tsvankin, I., 1997, Anisotropic parameters and P-wave velocity for orthorhombic media: Geophysics, 62, 1292-1309, doi: 10.1190/1.1444231.

Tsvankin, I., J. Gaiser, V. Grechka, M. Van Der Baan, and L. Thomsen, 2010, Seismic anisotropy in exploration and reservoir characterization An overview: Geophysics, 75, no. 5, 75A15-75A29, doi: 10.1190/1 .3481775 .

Van Trier, J., and W. W. Symes, 1991, Upwind finite-difference calculation of traveltimes: Geophysics, 56, 812-821, doi: 10.1190/1.1443099.

Vidale, J. E., 1990, Finite-difference calculation of traveltimes in three dimensions: Geophysics, 55, 521-526, doi: 10.1190/1.1442863.

Wang, C., D. Yingst, J. Bai, J. Leveille, P. Farmer, and J. Brittan, 2013, Waveform inversion including well constraints, anisotropy and attenuation: The Leading Edge, 32, 1056-1062, doi: 10.1190/tle32091056.1.

Wang, C., D. Yingst, R. Bloor, and J. Leveille, 2012, VTI waveform inversion with practical strategies: Application to 3D real data: 82nd Annual International Meeting, SEG, Expanded Abstracts, doi: 10.1190/ segam2012-0736.1.

Wild, P., and S. Crampin, 1991, The range of effects of azimuthal isotropy and EDA anisotropy in sedimentary basins: Geophysical Journal International, 107, 513-529, doi: 10.1111/j.1365-246X.1991.tb01412.x.

Wu, R., and K. Aki, 1985, Scattering characteristics of elastic waves by an elastic heterogeneity: Geophysics, 50, 582-595, doi: 10.1190/1.1441934. 